\title{
La terra di mezzo del commentario umanistico ai testi classici
}

\author{
Giancarlo Abbamonte \\ Università di Napoli 'Federico II' \\ giancarlo.abbamonte@unina.it
}

\begin{abstract}
Scholars have lengthy debated on the originality of the humanistic commentary on the classical authors with respect to the medieval commentaries on the same authors. If the question can be regarded as still open for the works written in the first half of the $15^{\text {th }}$ century, the birth of the printing determined a dramatic change in the contents and the form of the commentaries. From the point of view of the content the humanists are much more interested in the different readings transmitted by the manuscripts, whilst the printing allows both to have different layouts of the commentaries and to insert new tools as indexes and page numbers for consulting them. The present paper will present the new aspects of the printed commentaries and will try to explain the reasons which produced each change.
\end{abstract}

\section{Keywords}

Humanism - Frame-commentaries - Page numbering and Indexes in printed books

\section{1 È esistito un commentario umanistico? Le posizioni della critica recente $\left(1975^{-2013}\right)$}

Sebbene la domanda presente nel titolo del paragrafo possa apparire retorica, in realtà si tratta di una questione ancora sub iudice: da un lato è evidente che il periodo storico-culturale che va sotto il nome di Umanesimo ${ }^{1}$ produsse opere

1 Per comodità e con tutti i limiti di ogni delimitazione cronologica, si considererà qui il periodo incluso tra il 1397 (arrivo di Crisolora a Firenze) e il 1527 (sacco di Roma ad opera dei 
che rientravano nel genere del commentario; ${ }^{2}$ ciò non significa, però, che si possa senza problemi affermare che sia esistito un commentario umanistico con caratteristiche proprie che lo differenzino da quello che è trasmesso sin dall'epoca tardo-antica ${ }^{3}$ e che si è diffuso in tutto il corso del Medioevo, soprattutto grazie allo sviluppo dell'insegnamento basato sulla lettura dei testi (latini) e sul loro commento che si verificò a partire dal Basso Medioevo (XI-XIV secolo). ${ }^{4}$

In anni recenti ha espresso una posizione 'continuista' lo storico inglese Robert Black, il quale ha dedicato tre importanti saggi alla storia dell'insegnamento in Italia dal XII al XV secolo, nei quali ha applicato un metodo quantitativo, basato sulla ricerca d'archivio. ${ }^{5} \mathrm{Nel}$ suo primo lavoro, pubblicato insieme a Gabriella Pomaro, Black ha osservato, attraverso l'esame di documenti scolastici provenienti da archivi e biblioteche della Toscana, il persistente uso della Consolatio philosophiae di Boezio nell'insegnamento grammaticale del latino anche nel corso della prima metà del XV secolo, in cui Boezio non godette di buona stampa presso gli umanisti. ${ }^{6}$ L'esame di questa documentazione induce

Lanzichenecchi), cui saranno dati i nomi convenzionali di 'Umanesimo' e 'Quattrocento', mentre si eviterà la traduzione dell'espressione inglese Early Modern Age o del tedesco Frühe Neuzeit, che rimandano a periodizzazioni più ampie, funzionali a quelle aree europee, in cui l'Umanesimo arrivò molto dopo. Si segue qui la periodizzazione dell'Umanesimo discussa da Kristeller 1985, 93-95, che differenzia questo periodo dal più ampio concetto di 'Rinascimento' (1300-1600).

2 Dal punto di vista quantitativo il sito degli incunaboli della British Library (http://data.cerl. org/istc/_search) registra sotto il radicale comment* 985 titoli a stampa. Tuttavia, questi dati, che includono anche commenti medievali messi a stampa o commenti di testi non classici, necessitano di essere vagliati e interpretati.

3 Ad es., il commento di Donato alle commedie di Terenzio, quello di Servio alle opere di Virgilio o del cosiddetto Lattanzio Placido a Stazio.

4 Il problema fu sollevato anche da James Hankins e ripreso in un panel del 12th International Congress for Neo-Latin Studies (Bonn, 3-9 agosto 2003), confluito in Pade 2005, come ricorda la stessa studiosa in Pade 2005a, 3, vd. infra.

5 Vd. Black-Pomaro 2000, Black 2001 e Black 2007.

6 Il periodo esaminato da Black trova significative corrispondenze nelle tradizioni manoscritte di autori classici come Virgilio e di grammatici come Donato, i cui manoscritti esemplati tra VIII e XI secolo presentano caratteristiche differenti da quelli databili a partire dal XII secolo: vd. Holtz 1986, 127-128. Contro Boezio vd. Valla Eleg. vi 34 (ediz. di Basilea 1540), passo ricordato da Black 2001, 238. Sull'uso scolastico della Consolatio cfr. Black-Pomaro 2000, ma anche Black 2001, 236-238, che ricorda come Bartolomeo Fonzio adoperasse ancora la Consolatio nella Firenze di fine Quattrocento (ma dalla metà del secolo la presenza della Consolatio diminuisce: cfr. Black 2001, 270-271). Accanto a Boezio si continuarono ad adoperare fino alla metà del secolo anche i vituperati testi medievali minori: l'elenco in Black 2001, 226-227, e 272-273 per la loro eclissi alla metà del Xv secolo. Al di fuori della Toscana Boezio compare tra i testi scolastici per l'insegnamento del latino in alcuni monasteri francescani abruzzesi nel corso del XIV e XV secolo: vd. Raso 2001 e Abbamonte 2007. 
Black a concludere che il fenomeno dell'Umanesimo sia sopravvalutato dagli studiosi nei suoi effetti sulla storia dell'insegnamento del latino elementare impartito agli Italiani del XV secolo. Anche in quel secolo di grandi trasformazioni culturali, il latino (per chi aveva accesso alla scuola) fu insegnato non da pochi umanisti innovatori, ma dai molti anonimi docenti che perpetuavano metodi didattici, esercizi di apprendimento, manuali, autori da leggere e commentare e strategie di commento che si erano adoperati a partire dal XII secolo, quando si verificò la grande trasformazione dell'insegnamento del latino: tra questi metodi rientravano la lettura e il commento di una selezione, modificata nel corso dei secoli, ${ }^{7}$ di autori latini che intervenivano nella fase più avanzata dell'apprendimento del latino.

Black prende così le distanze dalla maggior parte degli studiosi precedenti dell'Umanesimo (filologi, storici e storici della filosofia), i quali sarebbero più attenti alle grandi individualità del Quattrocento che all'insegnamento corrente, testimoniato dalle rilevanze documentarie:

Another problem with modern scholarship on Italian curriculum history is that it tends to look from the top down. Dominant in previous writings have been the names of humanist luminaries such as Vergerio, Barzizza, Guarino, Perotti and Poliziano. This has no doubt been due to the prominence in this field of philologists, who are justifiably concerned with outstanding and innovatory individuals. ${ }^{8}$ The humdrum anonymous school grammarians, glossators or pupils have scarcely interested the students of humanist philology [...], it was now time to turn to the lower strata of culture in order to see how more than 99 per cent of the population actually learned Latin. ${ }^{9}$

7 Le differenti selezioni di autori da leggere intervenute tra XII e XV secolo sono esaminate nel quarto capitolo di Black 2001, 173-274 (Latin Authors in Medieval and Renaissance Italian Schools: the Story of a Canon). Per i primi livelli di insegnamento gli auctores e i testi letti restano costanti in questi secoli, mentre si osservano cambiamenti nella selezione degli autori che servivano alla parte più avanzata dei programmi che riguardava la stilistica latina. La lettura e il commento degli auctores erano preceduti dagli Accessus, su cui vd. infra.

8 Black 2001, 2, ricorda i lavori dedicati alla storia dell'Umanesimo e dell'educazione in Italia da Remigio Sabbadini (1902, 1922), Giuseppe Manacorda (1914) e Giuseppe Saitta (1928), o quelli successivi di Eugenio Garin $(1953,1957)$ e per restare più vicini ai nostri giorni gli studi di Carla Frova $(1973,1992)$ e Paul Grendler $(1989,1996)$ per citare i maggiori, che però non hanno cambiato il quadro delineato dai primi studiosi e da Garin.

9 Black 2001, 3. Su un piano più teorico giunge a conclusioni simili Zumthor 1990, in part. 12-15, che non vede discontinuità nel passaggio dalla glossa medievale alla critica letteraria dei commentari di età moderna. 
Black prende ad esempio le opere sulla sintassi dei casi scritte da famosi umanisti, come la Regule grammaticales di Guarino o i Rudimenta grammatices di Perotti, rilevando una mancanza di originalità nella struttura e nel metodo didattico rispetto alla precedente tradizione medievale:10

Guarino and Perotti were both famous Renaissance humanists, and so historians have felt either determined or at least obliged to discover some kind of significant innovation in their teaching of secondary grammar: since the Latin language was at the heart of the Italian Renaissance, there must have been some major upheaval associated with the approach of Quattrocento humanism to formal grammar in the schoolroom. This is yet another example of writing history backwards: because the teaching of secondary grammar underwent profound changes under the influence of Renaissance humanism, in the sixteenth and seventeenth centuries, especially in Northern Europe, it has been assumed that this reform movement was initiated and reached a significant stage of development in humanism's cradle, Italy, during the fifteenth century [...]. If the humanists were revolutionaries in the schoolroom, it was not in their formal grammar teaching."1

Le successive ricerche documentarie di Black hanno riguardato l'educazione a Firenze: da esse risulta confermata la lettura 'continuista' dell'organizzazione didattica in uso a partire dal XII secolo, e in particolare dell'insegnamento elementare del latino. ${ }^{12}$ Senza negare la funzione ammodernatrice degli umanisti italiani, Black l'ha limitata all'ultimo livello dell'insegnamento del

10 Le pretese di originalità degli umanisti sarebbero, secondo Black, solo finalizzate alla loro carriera: "It is misleading to take humanists' criticisms of the medieval heritage out of their ideological context. In Italy, these attacks were made not so much as a manifesto of a root-and-branch educational reform but rather as a platform for professional advancement, to secure a place for themselves at the higher levels of the educational hierarchy", Black 2001, 368 .

11 Black 2001, 124-125. Il ragionamento di Black si può estendere anche all'attività didattica di un altro umanista, Antonio Mancinelli (1452-1505), il quale fu un famoso insegnante in varie città d'Italia e pubblicò a stampa molte opere didattiche, tra cui alcune relative alle fasi iniziali dell'insegnamento del latino: il loro successo editoriale non si deve ad alcuna novità didattica, ché esse riproducono struttura e contenuto dei testi in uso dal XII secolo (la Ianua e un suo commento ai Disticha Catonis): Black 2001, 235. Su Mancinelli vd. Lazzari 2005 e Mellidi 2007; sulle sue opere didattiche di primo livello e le loro stampe: Gehl 2008, e Gehl Humanism for Sale, 3.06; sul ruolo di Mancinelli nello sviluppo dei commentari plurimi vd. infra 180-183.

12 Cfr. Black 2007, in part. XIII per la delimitazione delle fasi di insegnamento trattate. 
latino (selezione e lettura dei classici, studio della retorica), ${ }^{13}$ mentre un primo consolidamento degli ideali umanistici nella scuola è individuato da Black solo nell'ultimo trentennio del secolo, quando ebbero un ruolo decisivo in questo fenomeno l'introduzione della stampa e alcune scelte educative consapevoli fatte dalle classi dirigenti delle città. ${ }^{14}$ Tuttavia, i cambiamenti dell'ultimo trentennio del XV secolo non furono, secondo Black, profondi come la cesura rispetto alla tradizione didattica medievale che intervenne nell'Europa transalpina dei secoli XVI e XVII, con un contributo solo indiretto dei grandi umanisti italiani del secolo precedente. ${ }^{15}$

In definitiva, la prassi didattica non mutò fino all'ultimo trentennio del XV secolo, perché i valori educativi elaborati dagli umanisti già dalla fine del XIV secolo non erano penetrati nella scuola. In questa prospettiva, anche i commentari ai testi classici latini prodotti nel corso del xv secolo da autori che rientrano a pieno titolo nel movimento umanistico (ad es., Guarino), non dovrebbero differenziarsi da quelli dei secoli precedenti. ${ }^{16}$ Tuttavia, ogni conclusione rischia di essere azzardata per la produzione della prima metà del Quattrocento, in quanto le nostre conoscenze dei commentari di quel periodo sono ancora assai lacunose: manca a tutt'oggi un censimento dei commentari di autori classici prodotti in questo periodo e dei manoscritti che ce li trasmettono; è persino superfluo aggiungere che dei contenuti di questi commenti e delle loro relazioni con la tradizione medioevale precedente, in assenza di studi e di edizioni, non si può dire nulla di preciso.

Non si mostra in contrasto con i lavori di Black il progetto sul genere del commentario in età moderna portato avanti in anni recenti da Karl A. E. Enenkel e culminato con la pubblicazione del volume miscellaneo in collaborazione con Henk Nellen, Neo-Latin Commentaries and the Management of Knowledge in the Late Middle Ages and the Early Modern Period (1400-1700), Leuven 2013, e del successivo, curato dal solo Enenkel, Transformations of the Classics via Early Modern Commentaries, Leiden 2014, in cui sono esaminate varie

13 Black 2001, 238-274, per l'elenco dei classici, e 349-365, sulle innovazioni nel campo della retorica.

14 In Black 2001, 11, 269-273, e Black 2007, 462-468, è evidenziato l'interesse delle classi elevate di Firenze (upper rhetorical levels) verso l'educazione latina di tipo umanistico.

15 In varie parti del volume del 2001, Black considera l'introduzione della stampa una frattura, ma non insiste in proposito, poiché il tema esula dall'ambito cronologico delle sue ricerche: cfr. Black 2001, 270.

16 Le tesi di Black sono state fatte proprie da altri eminenti studiosi dell'Umanesimo, come James Hankins, su cui vd. Pade 2005a e la nota 4 supra. Emblematico è il caso preso in esame da Ulery 2005, su cui vd. infra, 163 . 
specie di commento e non solamente quelle legate all'insegnamento del latino. ${ }^{17}$ Sebbene i due volumi tengano conto di una periodizzazione che abbraccia l'intero XV secolo, nessun saggio tratta casi di commenti prodotti prima della seconda metà del Quattrocento: in generale, l'avvento della stampa è considerato l'evento principale per lo sviluppo delle diverse tipologie di Neo-Latin commentaries, ${ }^{18}$ come aveva già rilevato Black per l'insegnamento del latino. ${ }^{19}$

Prima degli studi di Black, Enenken e Nellen erano apparsi a partire dagli anni '7o del secolo scorso importanti lavori sul genere del commentario e sulla sua evoluzione dall'Antichità all'Età moderna, che hanno avuto il merito di precisare le diverse tipologie di questa para-letteratura ${ }^{20}$ e le applicazioni a numerosi generi di testi nel corso dei secoli. ${ }^{21}$ Alcune di queste opere, tutte miscellanee, si sono concentrate sul genere del commentario in Età moderna: nella maggior parte dei casi, queste raccolte sono dedicate a commentari nati in epoche successive a quella qui esaminata, quando le trasformazioni del commentario medievale grazie al progresso della stampa erano ormai un dato di fatto. È questo il caso del volume curato da R. Häfner e P. Völkel del 2006,22 che programmaticamente si concentra su commentari prodotti in piena Età moderna (XVI e XVIII secolo) e contiene molti saggi dedicati a testi non letterari. ${ }^{23}$

17 Il primo volume comprende cinque sezioni, che illustrano diverse tipologie di testi commentati: storia e geografia (I), poesia classica (II), teatro (III), diritto (IV), studi biblici (V); il secondo si divide in poesia (I), storia e filosofia morale (II), storia naturale e geografia (III).

18 Nel volume di Enenkel-Nellen 2013 non è usata l'espressione 'commentario umanistico', in quanto i commentari esaminati sono indicati in base alla lingua in cui essi furono scritti (Neo-Latin), mentre per l'arco cronologico considerato si parla di Early Modern Period, categoria ampliamente utilizzata in molti paesi europei: vd. supra nota 1.

19 Nella lunga introduzione Enenkel-Nellen 2013b, 1-76, Black è citato (vd. note 33 e 85), ma non mi sembra che siano discusse le sue conclusioni.

20 I concetti di para-letteratura e para-testualità e la stessa terminologia ancora in uso sono stati introdotti nel famoso saggio di Genette 1989 sulle varie parti di un libro che non costituiscono il 'testo': bisogna precisare che per lo studioso francese anche il commento rientrava tra i paratesti, mentre in questo lavoro esso sarà considerato testo a tutti gli effetti: vd. Genette 1989, 315-336, il capitolo sul concetto di nota, e le osservazioni di Enenkel 2014a, 3-4.

21 Sulla storia del genere del commento vd. i volumi curati da Most 1999, e GibsonShuttleworth Kraus 2002.

22 Vd. Häfner-Völkel 2006.

23 Vd. l'introduzione di Häfner-Völkel 2006, 1, scritta da Häfner: su otto contributi, due sono dedicati a commentari teologici, uno a testi storici, uno al Petrarca volgare e uno al neoplatonismo di Ficino; ai testi latini si rivolgono i lavori di Häfner sul commento di 
In alcuni lavori degli ultimi decenni, fa già la sua comparsa l'interrogativo posto da Black circa la continuità o meno dei metodi didattico-esegetici in uso tra XII e XV secolo. Così, di sostanziale continuità di istituzioni, metodi e tipologie di commentario parla August Buck nell'Introduzione alla miscellanea Der Kommentar in der Renaissance, curata dallo stesso Buck e da O. Herding nel 1975, che apre cronologicamente questa rassegna di studi dedicati al commentario (Buck 1975, 8):

Der Kommentar, der 'die wichtigste Form der gelehrten Literatur des Mittelalters dargestellt' und seit dem 12. Jahrhundert in allen Wissensgebieten Eingang gefunden hatte, 'wurde bis zum 16. Jahrhundert und darüber hinaus gepflegt'. ${ }^{24}$ Wie auch in anderen Bereichen zeigt sich hier eine Kontinuität [...].

Il volume di Buck e Herding mostra particolare attenzione ai commentari ai testi letterari latini, di cui rileva come gli ampliamenti intervenuti nel novero degli autori commentati e nelle finalità non abbiano mutato la loro destinazione, che restò il mondo dell'insegnamento. ${ }^{25}$ La tipologia di commentario esaminata in quest'opera ritorna nella più recente miscellanea sull'argomento, curata da Marianne Pade, dal significativo titolo On Renaissance Commentaries (2005), che contiene sei lavori su commentari ad opere di autori latini scritti tra il XV e gli inizi del XVI secolo. ${ }^{26}$ Nella premessa la curatrice ricostruisce le origini del volume nelle stimolanti osservazioni di James Hankins circa la possibilità o meno di individuare elementi distintivi nel commentario umanistico rispetto alla tradizione precedente - invero, i saggi contenuti nel volume non forniscono una risposta univoca a questo problema. ${ }^{27}$

Casaubon all'Apologia di Apuleio e quello di Blair 2006, sui Collective Commentaries, che discute la natura di opere come il Cornu copiae di Perotti, su cui vd. infra 172-175.

24 Citazione da Kristeller 1960, 215.

25 Conferma la continuità di testi commentati il lavoro di Desmet-Goethals 1975, nello stesso volume, relativo ai commentari di umanisti (Josse Bade Ascensius, Mancinelli, Erasmo e Corderius) ai Disticha Catonis, uno dei pilastri dell'insegnamento grammaticale medioevale.

26 Solo il saggio di Kallendorf 2005 si estende ai secoli successivi.

27 La natura 'aperta' del volume è sottolineata dalla recensione di Skoie 2006: all'interno della miscellanea la curatrice dedica il suo saggio al Cornu copiae di Perotti, la cui appartenenza al genere del commentario è problematica e si giustifica, secondo Pade 2005b, con le nuove finalità dell'insegnamento umanistico del latino volto a produrre non solo lettori, ma anche scrittori eleganti. 
Per il presente lavoro merita attenzione il saggio di R. W. Ulery, che tratta del commento al Bellum Catilinae di Sallustio attribuito all'umanista Ognibene Bonisoli in una stampa veneziana del $1500 .{ }^{28}$ Lo studioso dimostra che l'opera, attribuita falsamente ad Ognibene, era un anonimo commento medievale attestato per la prima volta in un manoscritto di XII-XIII sec. (Bern, Burgerbibliothek 411) e testimoniato da almeno sei manoscritti successivi. ${ }^{29}$ L'attribuzione ad un umanista fu opera dello stampatore, che volle dare così maggiore autorevolezza alla sua edizione. L'episodio è una conferma delle opinioni di Black, in quanto dimostra che anche per i lettori dell'epoca la differenza tra un commentario scritto trecento anni prima ed uno umanistico era talmente labile da permettere ad uno stampatore di attribuire ad Ognibene un testo medievale e perfino il suo Accessus senza timore di essere scoperto. ${ }^{30}$

Costituisce uno studio di ampio respiro teorico sul genere, corroborato da una vasta esemplificazione tratta da commentari di Età moderna, il volume collettivo francese Les commentaires et la naissance de la critique littéraire, curato da G. Mathieu-Castellani e M. Plaisance e apparso nel 199o: riassumendo i risultati dei diversi saggi ivi contenuti, i curatori intravedono uno sviluppo del genere dal XIV al XVI secolo attraverso una modifica della precedente glossa medievale, che fa da premessa alla grande stagione dei commenti di Età moderna. ${ }^{31}$

Questa breve historia critica consente di trarre qualche conclusione: tenuto conto di fisiologiche differenze di opinioni, esiste un certo accordo tra gli studiosi nel ritenere che l'eredità del commentario medievale abbia subito una trasformazione a partire dalla metà del XV secolo e che ad essa abbia contribuito l'avvento della stampa. Le novità riguardano sia gli aspetti esterni del commentario e il suo rapporto con il testo commentato (la mise en page, i paratesti, gli indici) sia i metodi esegetici addottati.

28 Vd. Ulery 2005. Si tratta della seguente stampa: Sallustius Crispus, Gaius, Catil. Comm. Laurentius Valla, Omnibonus Leonicenus; Iugurth. Comm. Johannes Chrysostomus Soldus; Invect. in Ciceronem; Excerpta ex libris historiarum; Add. Ps. Catilina, Oratio responsiva in Ciceronem, Corr. Pomponius Laetus et Johannes Britannicus, Venezia, Johannes Tacuinus de Tridino, 20.vir.1500, ISTC isooo8500o.

29 Cfr. Ulery 2005, 10.

30 In una stampa precedente è attribuito a Valla un commento a Sall. Catil., su cui vd. Osmond 2005 .

31 Cfr. Mathieu-Castellani e Plaisance 199ob, 7: sull'evoluzione della glossa in commento vd. i lavori di Zumthor 1990 e Jeanneret 1990, all'interno del volume. Questa ricostruzione è stata in parte corretta da Black 2001, 156 num. 665-668, che nota la coesistenza di differenti tipologie di glosse e commento sullo stesso manoscritto. 
Il presente contributo si colloca nel solco dei risultati raggiunti dai lavori summenzionati e cercherà di approfondire la natura dei commentari prodotti in quella terra di mezzo rimasta poco esplorata dalle ricerche precedenti, che si soffermano nella maggior parte dei casi sul commentario tardo medievale o su quello prodotto nella piena Età moderna fino al XVIII secolo, ${ }^{32}$ un periodo in cui il commentario fu un fondamentale strumento di trasmissione del sapere. ${ }^{33}$ In questa sede si esamineranno commentari prodotti nei decenni del primo sviluppo della stampa in Italia, per cercare di illustrare come alcuni fenomeni osservati per epoche successive siano già presenti nell'attività di alcuni umanisti italiani di quel periodo, che seppero cogliere le potenzialità di questo nuovo strumento di diffusione della parola scritta.

I documenti esaminati riguarderanno solamente i commenti ai testi classici latini, nella consapevolezza che le osservazioni che qui si presentano potrebbero non essere applicabili ai commentari rivolti ad altri tipi di testi, come ad esempio quelli filosofici o giuridici. ${ }^{34}$ Dei commenti ai testi letterari, trasmessi a stampa e/o su manoscritto, saranno messe in luce le novità dell'aspetto esterno (layout, novità paratestuali come gli indici) e del contenuto.

\section{$2 \quad$ Gli elementi esterni: mise en page, paratesti e indici}

Nel periodo in esame il commentario, sia su manoscritto che a stampa, trova spazio soprattutto nei margini del testo commentato, secondo una disposizione 'a cornice' (il cosiddetto frame-commentary), che prevede varie tipologie di rapporto tra testo e commento e la cui origine è disputata. ${ }^{35}$

Questa mise en page è documentata sicuramente a partire dal IX secolo nel mondo bizantino: tra i primi manoscritti con commentario a cornice si

32 Vd. il saggio di de Beer 2013, sull'aggiornamento geografico nei commenti ai testi di Plinio e Pomponio Mela intervenuto tra 1450 e 1700.

33 Sulla trasmissione del sapere in età moderna attraverso il commentario vd. EnenkelNellen 2013b, 21-29. In precedenza, aveva ben chiarito questa funzione anche Rigolot 1990.

34 Su questo punto l'autore desidera ringraziare gli studiosi che nel corso del dibattito seguito alla lettura della relazione hanno messo a confronto le loro esperienza di lettura di commenti a tipologie di testi differenti con quelle che sono state qui presentate.

35 Individua dieci tipologie di rapporto tra testo e commento Powitz 1979, ripreso da Maniaci 2002, 12 e 33; classificazioni dei documenti sono anche in Holtz 1984; per un approccio tipologico alla questione cfr. Sautel 1999, mentre un utile esame storico-documentario della glossa biblica è in de Hamel 1984. Gli scarsi dati a nostra disposizione rendono problematico stabilire una successione cronologica delle diverse tipologie: vd. Zetzel 1975, 339, 353-354, e Maniaci 2002, 11. 
ricordano un testo di Euclide di IX sec. (Vat. gr. 190) e alcuni famosi manoscritti di $\mathrm{x}$, forse dipendenti da una tradizione erudita risalente all'età imperiale: il Vat. Urb. gr. 35 di Aristotele, trascritto per Areta dopo il 902-903, ${ }^{36}$ il Venet. Marc. Graec. Z. 454 (= 822), noto come Venetus A di Omero; ${ }^{37}$ il manoscritto Paris. gr. 2771 di Esiodo, il manoscritto Firenze, BML Plut. 32,9 contenente Sofocle, Eschilo e Apollonio Rodio; il Ravennas 429 di Aristofane. ${ }^{38}$ Gli studiosi non hanno raggiunto un'opinione condivisa sull'origine di questa mise en page in ambito greco: secondo Zunzt, essa sarebbe stata sperimentata per la prima volta proprio intorno al IX secolo, favorita dall'introduzione della minuscola; Wilson e Irigoin ritengono, invece, che questi manoscritti di IX-X secolo riproducano modelli tardo-antichi e che la mise en page a cornice fosse in uso già a partire dal IV-V secolo. ${ }^{39}$

Anche nel mondo latino mancano testimonianze di commentari a cornice prodotti nella Tarda Antichità, mentre i dati a nostra disposizione sui commentari scritti in quell'epoca (Servio e Donato a Virgilio e Terenzio, Lattanzio Placido a Stazio, ecc.) sembrano confermare un layout a piena pagina, e non nei margini. ${ }^{40}$ I primi manoscritti che dispongono i commentari nei margini laterali contengono le opere di Virgilio e furono prodotti all'inizio del nono secolo in ambiente carolingio; la loro mise en page è ritenuta un'eredità della tradizione insulare. ${ }^{41}$ Gli studiosi non si esprimono sull'origine del fenomeno,

36 Maniaci 2002, 17-21, ritiene che la mise en page sia stata progettata da Areta stesso.

37 In questo manoscritto sono previste 25 linee per il testo di Omero e 63-70 per quello del commento marginale: cfr. Irigoin 1984, 99-101, Maniaci 2002, 21-22.

38 In questi manoscritti greci convivono commento marginale e glosse interlineari e le grafie di testo e commento sono spesso diverse tra loro (cfr. Irigoin 1984, 100), secondo quel sistema misto presente anche nei manoscritti scolastici latini: cfr Black 2001, 156, citato supra alla nota 31 .

39 Cfr. Zunzt 1938-1939; Irigoin 1984, 96-101 e Wilson 1984. In particolare, Irigoin 1984, 96-98, porta a sostegno della sua datazione il P.Oxy. 2258, testo di Callimaco con commento in margine (VI-VII sec., su cui vedi le perplessità di Maniaci 2002, 16 e nota 1), e l'uso all'interno degli scoli del verbo $\pi \alpha \rho \alpha ́ x \varepsilon ı \tau \alpha l$, che indica l'aggiunta in margine (vd. anche l'uso del verbo $\pi \alpha \rho \alpha \tau \imath \theta \varepsilon ́ v \alpha \iota$ in testimoni greci di IX-X sec., richiamato da Wilson 1984, 108).

40 Cfr. Maniaci 2002, 10-11 e le conclusioni di Zetzel 1975, il quale però sospetta l'esistenza di una versione ridotta del commento donatiano a Terenzio che si dispone nel margine e che fu adoperato dalla seconda mano che glossa il manoscritto Bembino di Terenzio (Zetzel 1975, 348-349). Nei margini del testo virgiliano si collocano gli Scholia Veronensia tramandati dal palinsesto XL (38) della Biblioteca Capitolare, tuttavia è possibile che essi siano stati aggiunti in un secondo momento su un manoscritto contenente le opere di Virgilio e non sembrano costituire un commentario continuo (ma la distinzione tra scoli e commentario continuo resta problematica): cfr. Baschera 1999, 43-44.

41 Cfr. Holtz 1984, 156-163. Si tratta dei famosi manoscritti Bern, Burgerbibliothek 165 (i cd. Scholia Turonensia, c. 820) e 172 (i cd. Scholia Bernensia e di Filargirio, c. 850). Analogo layout si ritrova in altri manoscritti virgiliani di IX sec. che dispongono nei margini il 
mentre convengono che la disposizione a cornice piena divenne abituale a partire dal Basso Medioevo con lo sviluppo del sistema scolastico. ${ }^{42}$

La maggior parte dei commenti manoscritti e a stampa del Quattrocento rispetta la struttura del frame-commentary nelle sue varianti: ${ }^{43}$ la forma più comune dispone il commentario su tre margini (superiore, esterno e inferiore), mentre la porzione di testo commentata, in corpo maggiore, è allineata all'interno (num. 2 Powitz $1979=$ Maniaci 2002, 33) [vd tavola 1]. ${ }^{44}$

Nel caso di commenti molto lunghi, lo specchio di pagina a cornice poteva essere interrotto da pagine prive di testo e con un fitto commento intercalato [vd tavola 2];45 in altri casi, per ragioni poco chiare, la forma a cornice era intervallata da una mise en page con commento nel solo margine esterno [vd. tavola 3$].{ }^{46}$ Più rara la mise en page con testo centrato e commento che si dispone nei quattro margini (num. 4 Powitz $1979=$ Maniaci 2002, 33) [vd. tavola 4]. ${ }^{47}$ Accanto a questa mise en page dominante, che si conserva dalla tradizione medioevale e che risponde ad esigenze di scuola, in cui era opportuno avere sotto mano testo e commento, cominciano a comparire elementi di novità nel rapporto tra testo e commento, che derivano dalle nuove possibilità fornite

commento di Servio: Hamburg, Staats- und Universitätsbibliothek, Scrin. 52, ma soprattutto Vat. Regin. Lat. 1669 (metà del IX sec.), in cui il commento di Servio trova posto in tutti i margini (cfr. Holtz 1985, in part. 27, e le tavole dell'Appendice finale di Holtz 1986). Enenkel-Nellen 2013b, 40. Ma vd. la nota precedente sui manoscritti anteriori all'xi secolo. A partire dal XII secolo la struttura a cornice penetra anche nella tradizione biblica (cfr. de Hamel 1984 e Maniaci 2002, 14-15) e in quella dei testi giuridici (Devoti 1999 e Maniaci 2002, 15).

43 Sulle tipologie di frame-commentary vd. supra nota 35 e Enenkel-Nellen 2013b, 39-40.

44 Numerosissimi gli esempi: per la poesia:Virgilio con il comm. di Servio, Venezia Antonius Bartolomaei, X.1486 (ma 1476), Istc ivoo1670oo; Ovidius, Heroid. (comm. A. Volscus), Sappho, Ibis (comm. D. Calderinus), Venezia, Lazarus de Suardis, 20.IV.1490, ISTC iooo156ooo; Ovidius, Fasti (comm. Ant. Constantius), Roma, Eucharius Silber, 23.X.1489, ISTC iooo17500o; Ovidius, Fasti (comm. di P. Marsus, ediz. curata da B. Merula), Venezia, Tacuinus de Tridino, 12.VI.1497, ISTC iooo176ooo; per la prosa i commenti di Francesco Maturanzio e Antonio Mancinelli alla Rhetorica ad Herennium, Venezia, Philippus Pincius, 8.viI.1496, ISTC icoo6830oo.

45 Cfr. Horatius, Opera (comm. Ps.-Acron, Pomponius Porphyrio, Christophorus Landinus), Venezia, Georgius Arrivabenus 4.II.149o/1491, ISTC iho04540oo; Cicero Off. (comm. Petrus Marsus), Lael. (comm. Omnibonus Leonicenus), Cato (comm. Martinus Phileticus), Parad. (comm. Jodocus Badius Ascensius), Lyon, Jean de Vingle, 30.VIII.1499, ISTC icoo61450o.

46 Cfr. Ovidius, Met. (comm. Rapahel Regius), Venezia, Bartholomaeus de Zanis, 27.II.14921493, ISTC iooo18700o.

47 Cfr. Horatius, Opera, Strasbourg, Johan (Reihnhard) Grüninger, 12.III.1498, ISTC ihoo46100o, in cui però solo qualche pagina rispetta questa mise en page, mentre nella maggior parte dei casi il commento si dispone sui margini laterali (si osservi in questa stampa anche l'uso delle glosse interlineari). 


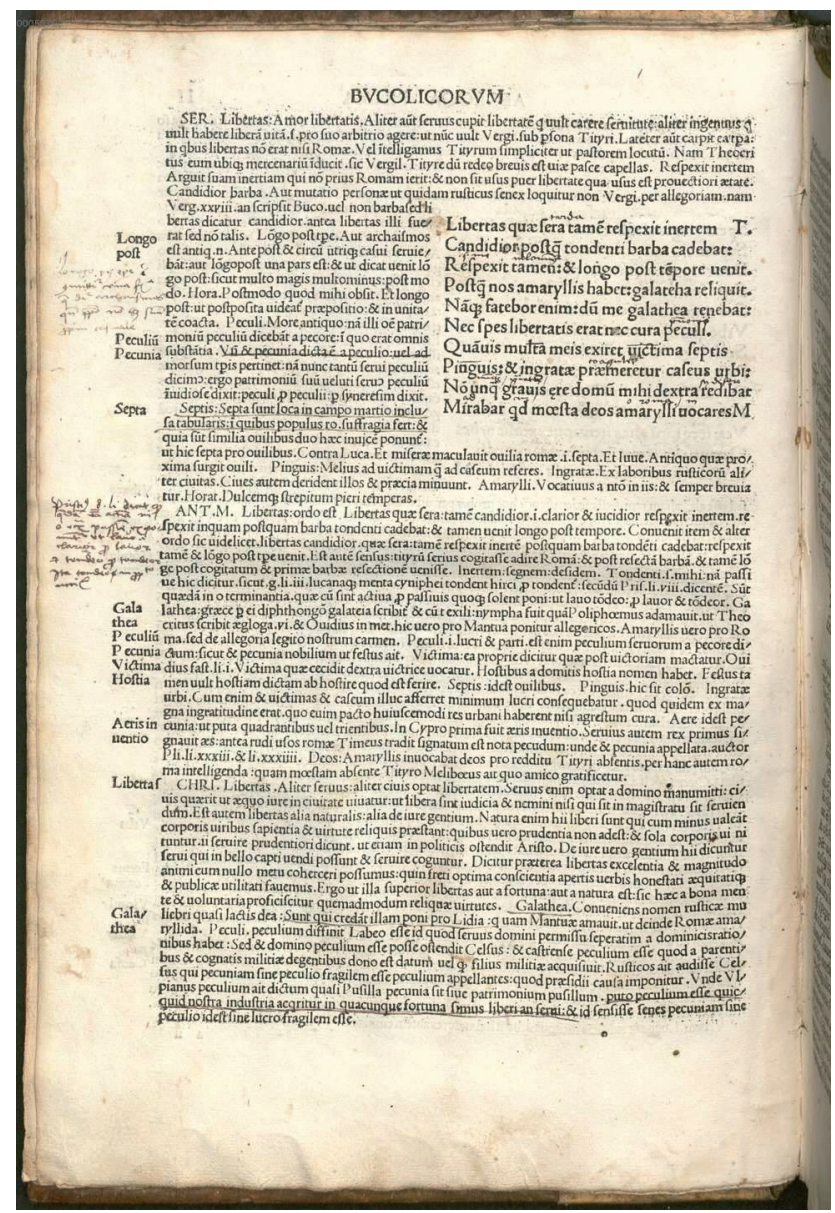

TAvola 1 Servio, A. Mancinelli, C. Landino, Commento alle Bucoliche $e$ alle Georgiche di Virgilio, Venezia, Philippus Pincius, 1491/2 ISTC ivoo188500.

dalla stampa. Nel primo commentario a stampa delle Silvae di Stazio, curato da Domizio Calderini (Roma 1475), ${ }^{48}$ i commenti sono collocati di seguito alla fine di ciascun poema e prendono la forma di un'epistola ad Agostino Maffei, che è anche il dedicatario dell'opera [vd. tavola 5$] \cdot{ }^{49}$ Il sistema trova applicazione

48 L'edizione fu stampata il 13.VIII.1475 a Roma da Arnold Pannartz (ISTC isoo6970oo) e contiene anche il commento di Calderini ad Ovidius Heroid. 15, all'epistola di Saffo a Faone, e la discussione di alcuni luoghi properziani.

49 Per questa tipologia di mise en page Enenkel-Nellen 2013b, 50 e 47 (figura 7), portano ad esempio il commento alle Odi di Orazio pubblicato da Dionysius Lambinus a Francoforte s. M. nel 1577, circa un secolo dopo quello di Calderini, e aggiungono che la novità di 

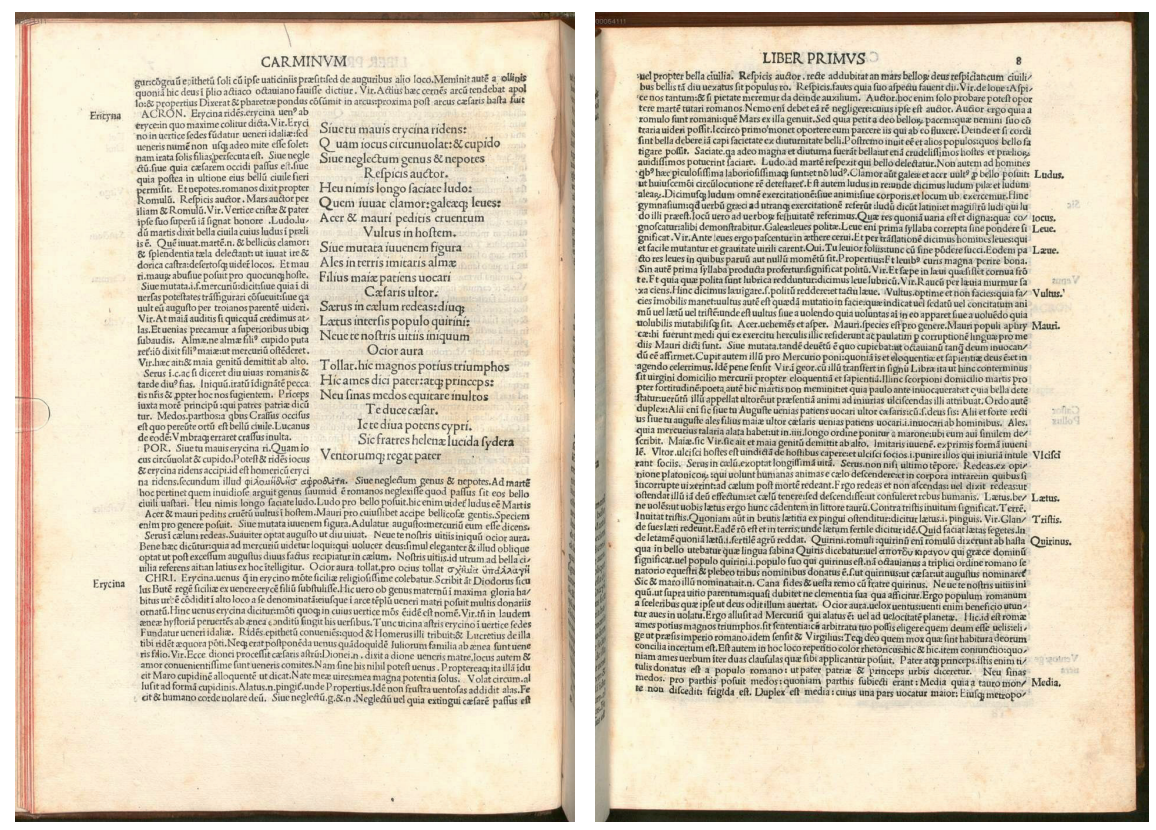

TAVola 2 Horatius, Opera (comm. Ps.-Acron, Pomponius Porphyrio, Christophorus Landinus), Venezia, Georgius Arrivabenus 4.II.149o/1491, ISTC ihoo4540oo.

anche per testi più lunghi delle Silvae come le satire di Persio: nell'edizione stampata a Lione da Jean de Vingle nel 1500 (ISTC ipoo361000), ogni satira è divisa in porzioni di versi, cui fanno seguito i commenti di Giovanni Britannico e di Josse Bade Ascensius.

Un'altra mise en page di cui Enenkel e Nellen attribuiscono la nascita all'apporto della stampa del XVI secolo prevede il commento indipendente dal testo, in modo che appaia in primo piano il ruolo del commentatore. ${ }^{50}$ L'origine di questa disposizione, che mette in risalto la personalità dell'umanistacommentatore, che si colloca alla pari se non al di sopra del testo commentato, è da anticipare alle prime stampe romane degli anni '7o del Quattrocento: la si ritrova nei Paradoxa in Iuvenali, commentario continuo delle satire di Giovenale scritto da Angelo Sabino $(1474)^{51}$ e in alcuni lavori esegetici di

questa mise en page, come di altre, sia frutto del progresso nei metodi di stampa avvenuto nel corso del XVI secolo.

5o Cfr. Enenkel-Nellen 2013b, 40, 48 (figura 8), 49 (figura 9) e 5o. Potrebbe essere letta in questi termini anche la vicenda sopra delineata (163) della falsificazione dell'autore del commento medievale a Sallustio, cui è attribuito il nome di un prestigioso umanista come Ognibene.

51 Roma, Georgius Sachsel et Bartholomaeus Golsch, 9.VIII,1474, ISTC isooo130oo. 
Tuncaderaselim miferiaque arra colebas.

Illud erattempusquore paftoria pellis

Texitonufque furt baculus filluct tris oliux

Alterius dif par feptenis phyftula cannis.

Durnque amor eft cura:dun te tua phyftula mulces

Incuftoditz pylios memoranturinagros

Procelfific boues.ruidet has atlantide maia

Natus:\& arte fua filuis occultat abactas.

Senferat hoc furtum nemo.nifi notus inillo

Rure lenex.battumuicinia tota uocabat.

Divitis hic faltus hxrbofaque pafcua nelei

Nobiliumque greges cuftos feruabatequarum.

Hunctimuit:blardaque manu feduxit:\& illi

$Q$ uilquises holpesait:fi forte armenta requirer

Hixcaliquistuidiffenega.neu gratra facto

Nulla rependatur:nitidam cape pramia uaccam.

Etdeditaccepta uoces has reddidit hofpes.

Tutus eas.lapisiffe prius tua furrta loquetur.

Et lapidem oftendit:fimulat loue natusabire.

Mox redit:\& ucrfa pariter cum uoce figura:

Ruftice uidiftifi quas hoclimite drxit

Ire boues:fer opem:furtoquefilentia deme.

Iuncta fuo pariter dabitur tibi formiua tauro.

At fenior poftä merces geminata:fübillis

Montibus inquitcrant:\& erant fubmontibusillis.

Rifitatlantiades:\& me mihuperfide prodis:

Me mihi prodis ait'́periuraq pectora uertit

In durum filicem:qui nunequoo; dicturindex:

Inque nihil merito uetusef infania faxo.

Hinc fe fiffulerat paribus caducifer alis.

Monichiofq; uolans agrostgratainq; minerus

Defpiciebar humum:cultiq arbufta lycei.

Illa fortediccaftrde more puella

Vertice fuppofito feftas in palladis arces

Pura coronatis portabant facracaniftris.

Indereuertentesdeus afpicitalessiterque

Non agit in rectú:fed in orbein curuateundem

Vtuolucris uifistapidiffima miluius extis

Dum timet:\& denfi circúftant facra miniftri:

Flectitur ingyrum:nec longius audetabıre:

Spemqiz fuamumotis auiduscircúuolat alis.

Spemqz fuam motis auiduscircúuolas

Sicfupcractzasas aglis cyllenius arces
Inclinat curfus:\& eafdem circinat auras.

$Q$ uanto f plendidior $\bar{q}$ cxtera fydera fulget

Lucifer:\& quanto ğlucifer aurea phobe:

Tanto uirginibus praftantior omnibus herfe

oritur unde Eleus pofféfiuum deriuatur.Vergliustertiogeo gicon. Hic uel ad Eleimetas \& maximacampisudabit ípacia urbs fuit grazciaz Pylo propin qua suxta quam Amphryfusia bitur. Alterius.dextre.Nam finiftra onus erat baculus. (I Abactasababductas. Propric Vnde abigidicunturarmenta rum fures 1 Nelei. Parer Neffo risfuit Neleus rex Pyli, o $\mathrm{Re}$ pendatur, referatur. \& Furto que filétia demehoc eft. furrú indica. (Atlantiades.Mercur us nepos Atlantisexmaiad Me irato conueniens finder lapis eft quo utimur ad aurumargé tumque probandum. (N) Nihi merito.Lapis enim mhil mal commifita, f Hincte fuftulerat, rom regis mutarionem in fuperionitranfformationicon nectit.Mercurius enim poft $\mathrm{B}_{3}$ tum in lapidem conuerrum for te inter uirgines Athenienfium facra mineruxe celebrátes uidit fierfen Cecropis iliam omnin potiri non poffet:Aglaurum il" lius fororem rogaurt:ut fibiopé Ferret.Eaoperam fuam poilice tur:dum ribi pecunia tribuat. Id cum relciuifict Pallas ob a Tamiamimmifit:qua impedita cum Mercurio repugnarer:ne Herfe amata frueret:in faxum ab co fuiteôuerfa. Munychios agrosaAiticos. Munychia nangrove dianza ICoronariscanifris. Canephoriaea facrauocata füt: quod in caniffris ferrentur.uir gines etiam Canephorx dice batur. Vetuolucris, Hac fimi litudinepoeta ret: ut diutius afpefou Herfes amatx frueret. (Miluiusauis eftrapax quze ingyrum uolat. (1) In gyrum in orbem $8<$ cie cuitum. (Circinat.circundat.

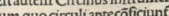
cum quo circuliaprecớticiunt,

TAvola 3 Ovidius, Met. (comm. Rapahel Regius), Venezia, Bartholomaeus de Zanis, 27.II.1492-1493, ISTC iooo187000.

Domizio Calderini, come i commenti all'Ibis di Ovidio (1474), ${ }^{52}$ all'Appendix ps. virgiliana (c. 1480);53 anche l'editio princeps del commento calderiniano a Marziale presenta questa mise en page (1474), ${ }^{54}$ mentre le stampe successive

52 Roma, Georgius Sachsel et Bartholomaeus Golsch, 7.IX.1474, ISTC icooo40000, edito da Rossi 2001.

53 Milano, Simon Magniagus?, c. 1480, ISTC icooo39ooo.

54 Roma, Johannes Gensberg, 22.III.1474, ISTC iсоооз6ooo. 


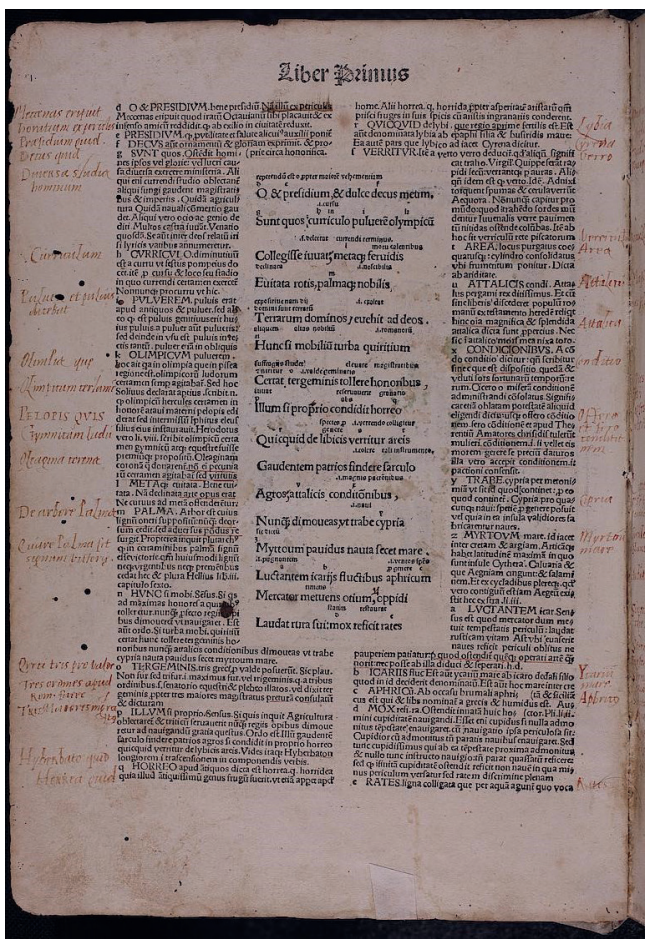

\section{TAVOLA 4}

Horatius, Opera, Strasbourg, Johan (Reihnhard) Grüninger, 12.III.1498, ISTC ihoo4610oo.

\section{adottano la forma a cornice, ${ }^{55}$ che si ritrova nella princeps del suo commento a} Giovenale (1475) e nelle ristampe. ${ }^{56}$

55 Mise en page a cornice in dieci ristampe controllate online: Venezia, Johannes de Colonia et Johannes Manthen, post 22.III.1474, ISTC icooo3700o; Venezia, stampatore del Marziale, 1480, ISTC imo304400o; Venezia, Thomas de Blavis, 12.VI.1482, ISTC imoo30500o; Venezia, Baptista de Tortis, 15.VI.1482, ISTC imoo306ooo (solita cornice a tre margini, ma anche solo nei due margini laterali), Venezia, Baptista de Tortis, 17.VII.1485, ISTC imo0308000 (identica impaginazione della precedente emissione); Milano, Uldericus Scinzenzeler, 20.IX.1490, ISTC imoo309ooo; Venezia, Philippus Pincius, 29.III.1491, ISTC imoo310ooo; Venezia, Bartholomaeus de Zanis, 13.XI.1493, ISTC imoo311000; Venezia, [Christophorus de Pensis], 1.VIII.1495, Istc imoo31200o; Venezia, [Christophorus de Pensis], 29.V.1498, ISTC imoo313000.

56 Venezia, Jacobus Rubeus, 24.IV.1475, ISTC ijoo64200o. Il dato si conferma in dieci delle 24 ristampe che si sono verificate online: Vicenza, Henricus de Sancto Ursio 1480, ISTC ijoo6440oo; Venezia, Baptista de Tortis, 31.X.1481, ISTC ijoo64500o; Venezia, Baptista de Tortis, 3.VIII.1482, ISTC ijoo646ooo; Milano, Leonardus Pachel et Uldericus Scinzenzeler, 16.IV.1483, ISTC ijoo6470oo; Venezia, Baptista de Tortis, 22.VII.1483, ISTC ijoo6490oo; Venezia, Baptista de Tortis, 30.III.1485, ISTC ijoo6500oo; Milano, Antonius Zarotus, 27.VI.1485, ISTC ijoo650500; Venezia, Bartholomaeus de Zanis, 3.X.1487, ISTC ijoo652000; 


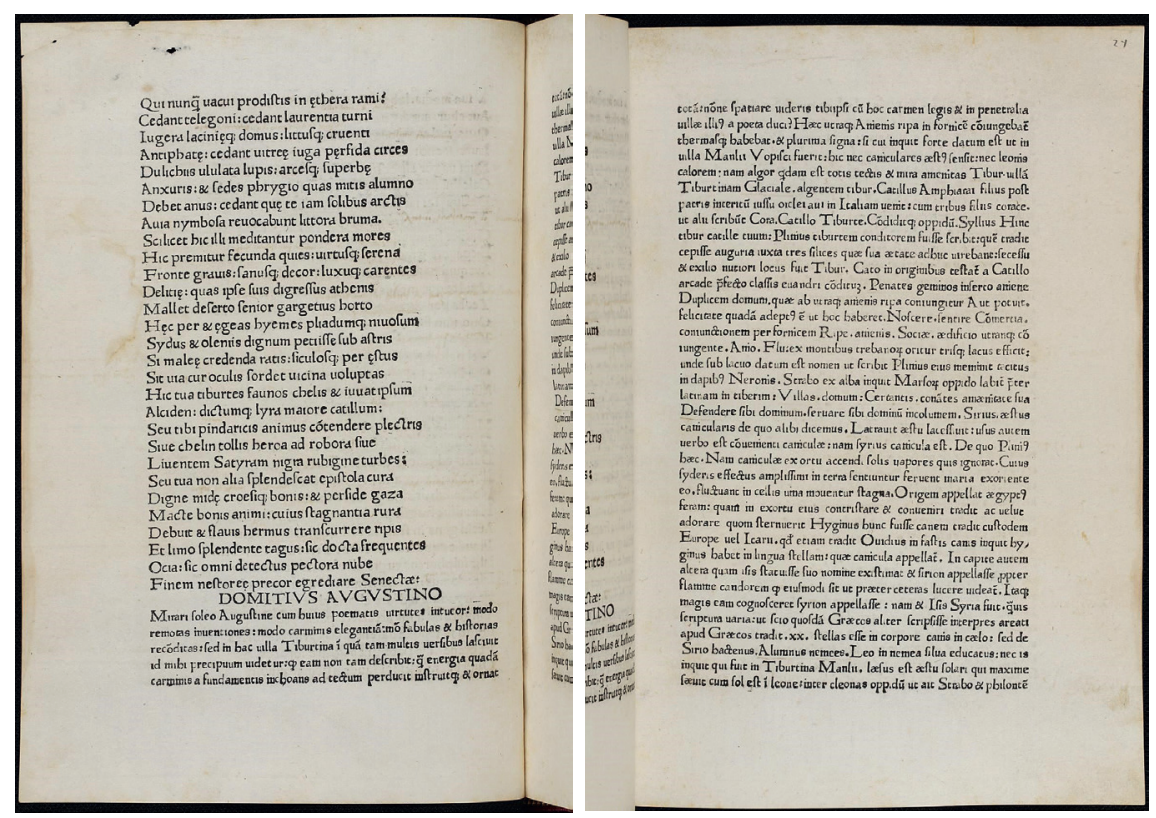

TAvola 5 Statius, Silvae (comm. di D. Calderini) Roma, Arnold Pannartz, 13.VIII.1475, ISTC isoo69700o.

In alcuni manoscritti coevi dei commenti di Calderini, progettati con la supervisione dell'umanista, si registra un'analoga mise en page, in cui il commento si accampa a pagina piena, mentre scompare il testo dell'auctor latino: tale disposizione si ritrova nel manoscritto del commento a Marziale offerto da Domizio Calderini a Lorenzo dei Medici nel 1473 (Firenze, BML, Plut. 53,33), e in quello del commento a Giovenale, che Calderini presentò a Giuliano dei Medici forse durante un suo soggiorno a Firenze nel settembre del 1474 (Firenze, B ML, Plut. 53,2); ${ }^{57}$ analoga disposizione si ritrova in un altro manoscritto di dedica a Lorenzo dei Medici, il commento di Bartolomeo Fonzio a Persio (Firenze, B ML, Plut. 54,23) e nel Vat. Urb. lat. 360, contenente il commento ai Fasti di Ovidio offerto da Antonio Costanzi a Federico da Montefeltro (1480). La disposizione a piena pagina può rispondere anche ad esigenze di insegnamento dell'umanista, come sembra indicare il manoscritto di Oxford, Bodleian. Canon. Class.

Milano, Uldericus Scinzenzeler, 30.viII.1488, ISTC ijoo65350o; Venezia, Theodorus de Ragazonibus, 16.VI.1491, ISTC ijoo6570oo.

57 Il periodo di composizione e la data di presentazione di questo manoscritto sono problematici: vd. Abbamonte 2015, 184-186. Si osservi che in questo caso le stampe non hanno rispettato la mise en page del manoscritto di dedica, che probabilmente era stata indicata dallo stesso Calderini. 
Lat. 54, che contiene il commento di Pomponio Leto alle opere di Virgilio e che è comunemente considerato una trascrizione di lezioni di Leto effettuata da uno studente ${ }^{58}$ tale disposizione si ritrova nelle Recollectae di lezioni di G. Pontano su Virgilio, Ovidio e Valerio Massimo, contenute nel manoscritto 1368 (T. 5. 5) della Biblioteca Angelica di Roma. ${ }^{59}$

Il layout a pagina piena esprime una rivendicazione di autorialità, che spiega anche la frequente presenza di polemiche all'interno dei commentari tardo-quattrocenteschi: si tratta di una cifra di novità, spiegabile con le forti personalità degli umanisti che si dedicarono a questi commentari e con la loro consapevolezza che i commenti avrebbero avuto una diffusione ben più larga delle aule scolastiche. Tra i commentari a pagina piena potrebbe rientrare anche il Cornu copiae di Niccolò Perotti (1430-1480), in quanto nel manoscritto che ce lo trasmette - in parte autografo e nato come esemplare di dedica al Duca di Urbino, poi forse trasformatosi in copia da lavoro (Vat. Urb. lat. 301$)^{60}$ - si legge il seguente titolo:

\section{NICOLAI PEROTTI CORNV CO-| PIAE SIVE COMMENTARIO-| RVM LINGVAE LATINAE AD | ILlVSTREM PRINCIPEM FEDE-| RICVM VRBINI DVCEM ET EC-| CLESIASTICI EXERCITVS IM-| PERATOREM INVICTISSIMVM | LIBER PRIMVS (f. 4V = p. 20 Charlet-Furno). ${ }^{61}$}

Induce ad inserire il testo nel genere del commentario non solo la presenza del termine Commentariorum nel titolo: l'opera si apre con il lemma «BARBARA PYRAMIDVM » (f. $4 \mathrm{r}=$ p. 20,1 Charlet-Furno), le prime parole del Liber de spectaculis di Marziale, di cui Perotti fornisce una lunga esegesi. Tutto il resto del Cornu copiae procede nella forma di un ipertrofico commentario continuo a piena pagina degli epigrammi di Marziale, di cui il manoscritto riporta solo i lemmi. Nonostante questi elementi, la natura del Cornu copiae resta difficile da determinare, forse perché l'autore mutò le sue intenzioni in corso d'opera e quello che in origine era stato immaginato come un commentario, assunse

58 Sul manoscritto di Fonzio e sul suo commento a Persio vd. Rossetti 2017, XIV-XVI, che ne mette in luce la natura scolastica; sul manoscritto di Costanzi vd. Toscano 2017, 63-75; sulle recollectae virgiliane di Leto contenute nel manoscritto di Oxford vd. infra nota 113 . Si osservi che anche i lavori di Calderini nascevano in margine alla sua attività didattica.

59 Vd. Iacono 2005, 15-30 per la descrizione del manufatto, che però non costituisce le originali recollectae, ma una loro copia eseguita da un erudito (Iacono 2005, 24).

6o Lo esamina come un commentario nel suo saggio Enenkel 2014a, 2-3, ma cfr. anche Pade 2005b e Pade 2013. Cfr. anche il titolo (nota 61).

61 'Primo libro del Cornu copiae di Niccolò Perotti o dei commentari della lingua latina, dedicato all'illustre principe Federico, duca di Urbino e invincibile generale dell'esercito pontificio'. 
la funzione di Commentarius linguae Latinae, cioè di vocabolario della lingua latina. ${ }^{62}$

Il Cornu copiae fu considerato il primo vocabolario moderno della lingua latina e per questa ragione godette di una straordinaria fortuna fino alla metà del Cinquecento; ${ }^{63}$ tuttavia, fu proprio la sua informe e ipertrofica struttura a richiedere l'introduzione di indici nelle sue edizioni a stampa. La mancanza di un ordine alfabetico rendeva impossibile la consultazione a chi volesse notizie su un singolo vocabolo. Per questo motivo, già nell'editio princeps del $1489,{ }^{64}$ lo stampatore e/o forse il curatore dell'edizione, Ludovico Odasi, avevano previsto un lungo indice alfabetico prima del testo: trenta pagine in folio divise in cinque colonne, che contenevano ciascuna all'incirca sessanta vocaboli [vd. tavola 6]. A partire dalla princeps tutte le altre edizioni del Cornu copiae furono precedute da indici che incrementarono il numero dei lemmi. ${ }^{65}$

Gli studiosi hanno da tempo evidenziato l'importante novità rappresentata da questi indici dei lemmi, che si perfezionano con l'invenzione della stampa e rivelano una nuova modalità di lettura dei libri: meno noto è che essi furono all'inizio collegati alla stampa del Cornu copiae. ${ }^{66}$ Probabilmente, quest'opera non fu considerata un testo da leggere per intero, come accadeva per i commentari tradizionali, nati spesso in margine a letture scolastiche e integrali dell'opera antica. Il capolavoro di Perotti perse presto, e forse già nella mente dell'autore, la sua natura di commentario per assumere quella di dizionario da consultare per singoli lemmi: questa nuova finalità determinò un progetto tipografico che rispondeva alle esigenze del più variegato mondo moderno, fatto non solo da studenti e docenti, ma anche da chi praticava il latino e aveva bisogno di compulsare un buon dizionario di riferimento per avere risposte su dubbi linguistici intervenuti sul lavoro (ad es. la stesura di un testo latino), nello studio, per interessi culturali o semplice curiosità. A conferma di questo nuovo modo di leggere, testimoniato dalle stampe del Cornu copiae, Enenkel

62 Il Cornu copiae condivide aspetti del lessico, dell'enciclopedia e del commentario: cfr. Pade 2005, 2005b e 2013, e Abbamonte 2005.

63 Vd. Milde 1982.

64 Venezia, Paganinus de Paganinis, 14.V.1489, ISTC ipoo2880oo.

65 Ad es., l'edizione stampata a Basilea da Valentinus Curio nel 1526 premette al testo perottino sei pagine in folio a cinque colonne di un indice di termini greci e 135 pagine a cinque colonne dell'indice dei termini latini. Di ogni item è fornito il numero di colonna e di linea: vd. Enenkel-Nellen 2013b, 55 .

66 Il ruolo del Cornu copiae non è rilevato in alcun lavoro raccolto da Leonardi 1995, un volume miscellaneo interamente dedicato alla storia degli indici, che però per contrappasso manca di indici. 


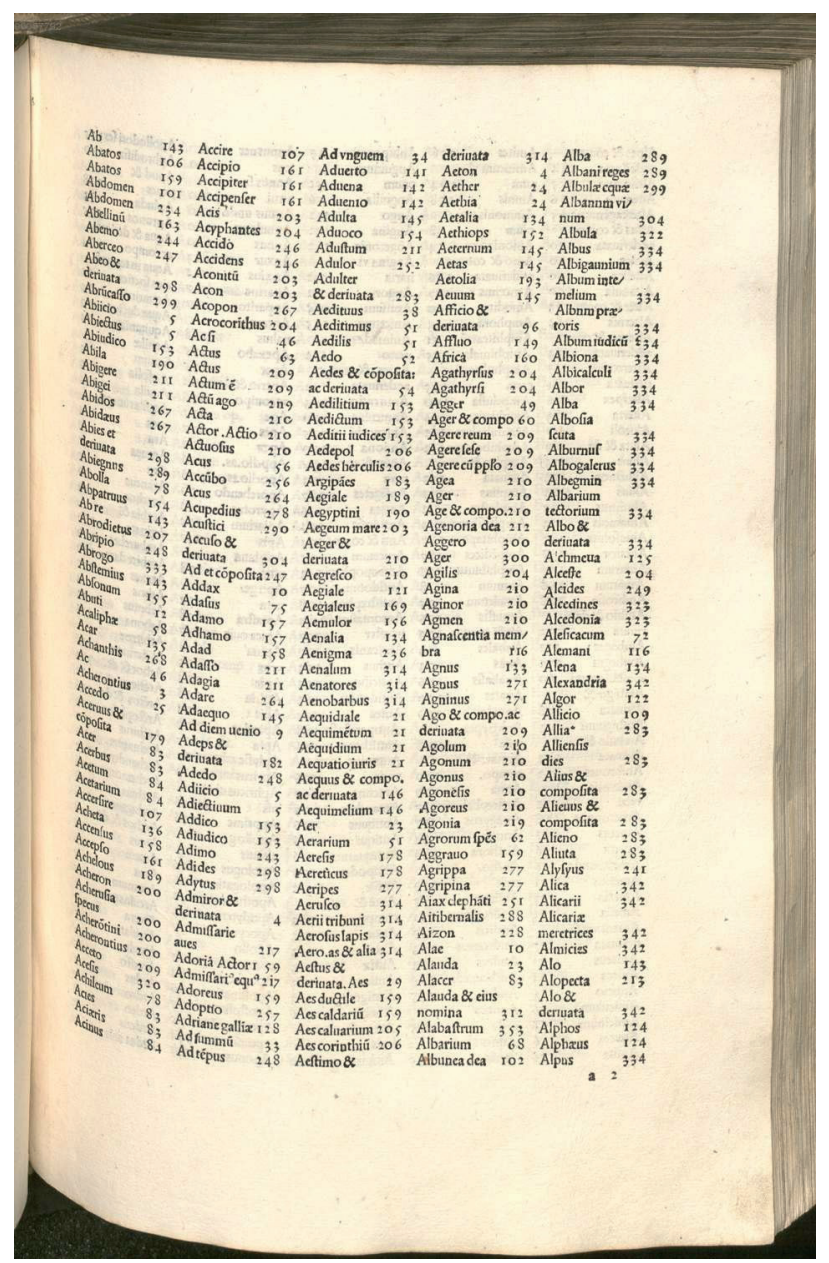

tavola 6 N. Perottus, Cornu copiae, Venezia, Paganinus de Paganinis, 14.V.1489, ISTC ipoo2880oo.

e Nellen mettono giustamente in rilievo il fatto che gli indici siano collocati prima del testo di Perotti, di cui costituiscono quasi la chiave di consultazione. ${ }^{67}$

Accanto agli indici, e loro necessaria precondizione, nasce il moderno sistema di numerazione delle pagine: nei manoscritti medievali spesso è assente qualsiasi numerazione e al massimo troviamo un'indicazione del numero di folio o di libro in testa alla pagina; nei primi incunaboli domina il criterio di numerare i fascicoli; nelle edizioni del Cornu copiae si sperimentano varie forme di numerazione fino ad arrivare alla numerazione delle pagine che permette il rapido reperimento dei lemmi indicizzati. L'editio princeps di Odasi

$67 \quad$ Enenkel-Nellen 2013, 54-57. 
aveva adottato il criterio di ripetere il numero di ciascun folio sul recto e sul verso; le successive edizioni veneziane lasciarono solo il consueto numero di folio sul recto ${ }^{68}$ fino alla rivoluzionaria edizione Aldina del 1499, che numera le singole pagine e aggiunge il numero di rigo sul margine interno di ciascuna pagina, per poi riportare il doppio dato in ogni lemma elencato nelle 51 pagine di indice premesse al testo ${ }^{69}$ [vd. tavola 7].

Un altro elemento paratestuale, che testimonia l'esistenza di un nuovo tipo di pubblico che legge e compulsa i commentari fa la sua comparsa allinterno del genere: le raccolte di Variae lectiones, dette anche Castigationes, Animadversiones, Exercitationes e Variae adnotationes. Come hanno ben evidenziato Enenkel e Nellen, questa sorta di indici tematici elencano i loci critici discussi all'interno del commentario rivelando un'attenzione verso il testo e i problemi filologici posti da esso. La cura verso il testo costituisce una novità dell'Umanesimo italiano che si ritrova nei commentari umanistici, diversi

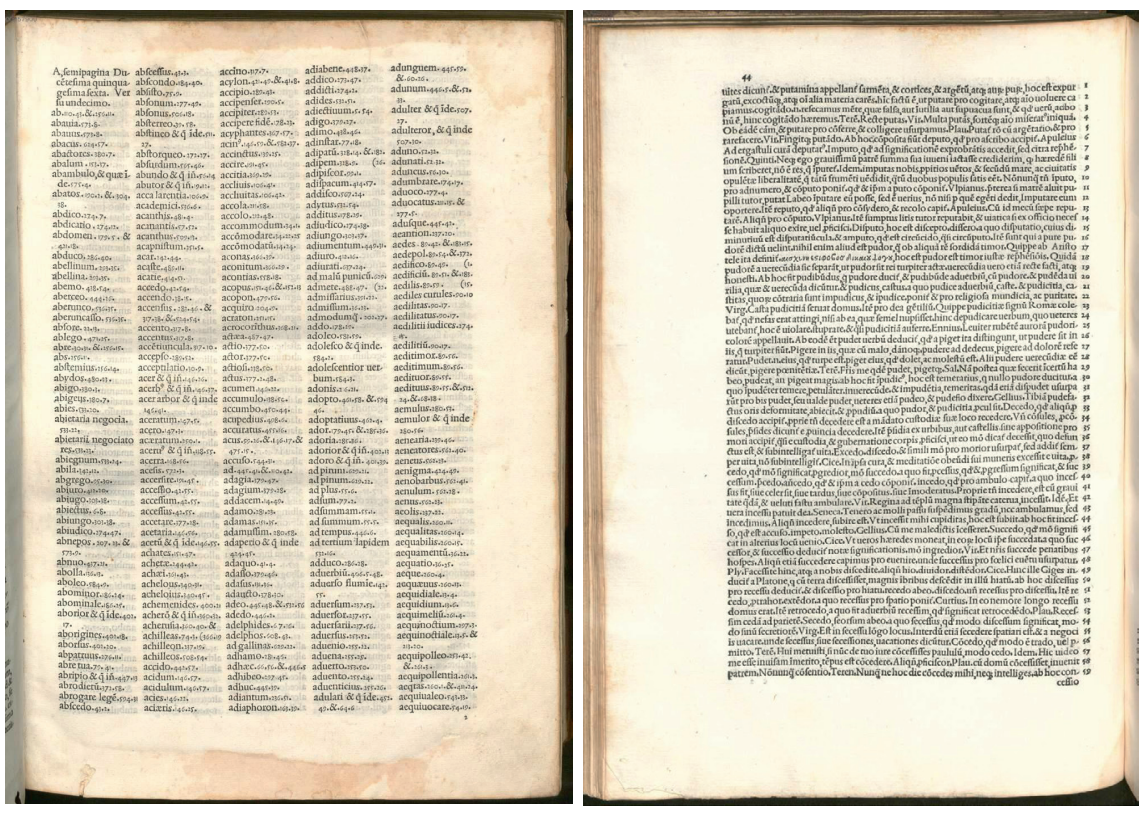

TAvola 7 N. Perottus, Cornu copiae, Venezia, Aldus Manutius, VII.1499, ISTC ipoo296ooo.

68 Venezia, Bernardinus de Choris, 30.V.149o, ISTC ipoo289ooo; Venezia, Baptista de Tortis, 19.X.1490, ISTC ipoo29oooo; Venezia, Bernardinus de Choris, 25.V.1492, ISTC ipoo291000; Venezia, Philippus Pincius, 27.III.1494, ISTC ipoo2920oo; Venezia, Dionysius Bertochus, 12.V.1494, ISTC ipoo2930oo; Venezia, Johannes Tacuinus de Tridino, 20.XII.1496, ISTC ipoo295000.

69 Venezia, Aldus Manutius, VII.1499, ISTC ipoo2960oo. 
anche da questo punto di vista rispetto a quelli medievali. ${ }^{70}$ Tali sezioni, poste per lo più alla fine del volume, si sviluppano soprattutto nel XVII e XVIII secolo: in esse si elencano gli interventi emendatori che erano tradizionalmente dispersi all'interno del commento o i risultati di collazioni effettuate su codici che fino a quel momento non erano stati presi in considerazione dagli editori. ${ }^{71}$ Anche in questo caso, alcuni lavori del tardo Quattrocento hanno inaugurato tale consuetudine: tra essi è famoso il commento di Ermolao Barbaro alla Naturalis historia di Plinio, dedicato alla discussione di loci critici, che recava il significativo titolo di Castigationes Plinianae (1492).

Queste raccolte di loci critici rivelano un lettore interessato a vedere riuniti questi dati filologici in una sezione specifica; questo interesse doveva essere tanto diffuso nel pubblico da indurre il commentatore e lo stampatore a preoccuparsi di predisporre tali sezioni. Quanto al pubblico che fruisce di queste sezioni, esso non è identificabile con il consueto ambiente scolastico del commentario: si tratta di raffinati studiosi di umanità in grado di apprezzare e discutere l'ingenium emendandi del commentatore o la sua capacità di scoprire lezioni originali nei codici.

In alcuni casi, queste raccolte raggiunsero l'autonomia di veri e propri volumi di Animadversiones, in cui erano raccolte le proposte emendatorie su vari autori avanzate da un singolo umanista: noti per la loro mole sono i sessanta libri di Adversaria pubblicati da Kaspar von Barth a Francoforte s. M. nel 1624. ${ }^{72}$ Anche la genesi di questa forma autonoma di raccolte di emendamenti a varie opere dell'Antichità trova le sue prima manifestazioni alla fine del Quattrocento nelle Emendationes in Plinium et Virgilium del Merula (Venezia, Johannes de Colonia et Johannes Manthen, c. 1474, ISTC imo0504000), ${ }^{73}$ le Observationes di Domizio Calderini, apparse in coda al suo commento alle Silvae di Stazio (Roma, Arnoldus Pannartz, 1475, ISTC isoo6970oo), in cui, come ricorda l'autore nel frontespizio, [...] nonnulla explicata sunt quae ad linguae latinae rationem magnopere pertinent, seguite dalle Annotationes centum di F. Beroaldo il Vecchio (Bologna, Franciscus de Benedictis, 1482 ISTC iboo4640oo), che furono

$70 \quad$ Enenkel-Nellen 2013b, 29-31, 57 e 61.

71 Vd. ad es., l'edizione delle opere di Tacito, in due volumi, con i commenti di G. Lipsio, B. Renano, F. Orsini, M.-A. Muret, etc. Amsterdam, Elsevier, 1672, che alla fine del secondo volume riporta otto pagine di Excerpta ex variis lectionibus MS. Oxoniensis, curate da J. Gronovius.

72 Enenkel-Nellen 2013b, 31, ricordano le Lectiones antiquae di Caelius Rhodiginus (Venezia, Aldus Manutius, 1516), gli Animadversa di Hadrianus Junius (Basilea, Michael Isengrinus, 1556) e le Variae lectiones di Giusto Lipsio (Anversa, Christopher Plantin, 1569).

73 L'opera compare in un volume miscellaneo che contiene di Merula l'In librum de homine Martii Galeotti opus, le Epistolae, l'In Sapphus epistolam interpretatio. 
riunite alle Observationes di Calderini in due ristampe successive. ${ }^{74}$ Una breve raccolta di luoghi del romanzo di Apuleio e delle Silvae di Stazio fu premessa da Giovanni Britannico al suo commento a Giovenale, ma i capolavori umanistici di questo genere sono rappresentati dai Miscellanea di Poliziano e dalla raccolta di loci critici in forma epistolare del De rebus per epistolam quaesitis di Aulo Giano Parrasio (c. 1521), pubblicati postumi. ${ }^{75}$

Come si è già osservato, se le raccolte dei loci critici rappresentano una novità e producono un ulteriore sviluppo delle sezioni paratestuali del commentario, ragionamenti sulle lezioni tramandate sono abitualmente presenti, ben prima dell'introduzione di questo tipo di paratesto, anche in commentari nati con finalità scolastiche, come dimostrano i lavori dell'umanista e stampatore Giovanni Britannico (c. 1448-1519), il quale rappresenta bene la generazione degli umanisti a cavallo tra XV e XVI secolo. ${ }^{76}$ Dopo una giovanile formazione sui testi classici Britannico spese la sua esistenza a Brescia dividendosi tra la stamperia di famiglia e le aule di insegnamento: i suoi testi, per evidenti ragioni commerciali, erano destinati in prevalenza al mercato scolastico bresciano; ${ }^{77}$ nondimeno, compaiono in essi discussioni filologiche, come si osserva nel suo commento a Persio, in cui Britannico interviene sui vv. 9-10 della seconda satira (o si ebulliat patruus, 'possa bollire lo zio'): ${ }^{78}$

EBULLET hunc ego locum emendavi. Nam cum omnes codices temporum fortasse et librariorum culpa 'ebulliat' legant, animadverti 'ebullet' legendum esse, non 'ebulliat'. Nam 'ebullio' secundam habet syllabam

74 Venezia, Baptista de Tortis, 1490-93, ISTC isoooo6ooo, e Brescia, Bernardinus de Misintis per Angelum Britannicum, 1496, ISTC iboo4650oo.

75 Su questo genere ancora utile Dionisotti 1968; sulle Observationes di Calderini cfr. Campanelli 2001, su Beroaldo vd. ora Severi 2015. Le Annotationes quaedam Ioannis Britannici in asinum Apuleii et Sylvas Statii compaiono ai ff. a ii-a iii del suo commento alle satire di Giovenale, Brescia, Ang. e Jac. Britannicus, 1501, ISTC iJoo66650o. Sull'opera di Poliziano la bibliografia è enorme: l'edizione di riferimento della centuria seconda è ancora quella di Branca - Pastore Stocchi 1972, mentre il De rebus per epistolam quaesitis di Aulo Giano Parrasio fu pubblicato nel 1567 da Henri Estienne: vd. la recente edizione critica di Ferreri 2012, in part. LXXXVII-XCII.

76 Su Giovanni Britannico vd. ora Rossetti 2017, XVII-LX.

77 Elementi che ne denotano l'origine scolastica oltre che la destinazione sono esaminati da Rossetti 2017, LXXX-LXXXVIII.

78 Il commento a Persio ebbe un'editio princeps pubblicata a Brescia da Gabriele di Pietro e suo figlio Paolo il 14.XI.1481 (ISTC ibo121300o), una prima revisione nell'edizione stampata a Brescia dal fratello Giacomo il 17.II.1486 (ISTC ipo03500oo) e una terza stampata ancora da fratello il 21.VII.150o (ISTC ipoo35100o): cfr. Rossetti 2017, LXII-LXIII, CXIV-CXXI, CLIII-CLVI. 
longam et geminato 'l' scribitur, ut alibi Persius: « Summa rursus non bullit in unda » $(3,34)$. Hoc loco vero si 'ebulliat' legatur corriperetur. ${ }^{79}$

Secondo l'umanista bresciano il testo andrebbe corretto per ragioni metriche, anche se la tradizione manoscritta è unanime nel tramandare ebulliat, come ammette lo stesso Britannico: ${ }^{80}$ nel passo si nota anche l'uso del verbo animadverto in un significato già tecnico, da cui sarebbe nato il genere letterario filologico delle Animadversiones. ${ }^{81}$

Anche se la maggior parte degli emendamenti proposti dagli umanisti del Quattrocento sono prodotti ope ingenii, non mancano a partire dal Cinquecento casi di emendatio ope codicum e perfino di umanisti che probabilmente hanno finto di aver trovato un emendamento in un manoscritto, come avviene in un gustoso episodio che riguarda il commento alla Poetica di Francesco Robortello (1548)..$^{82}$ Il passo in questione della Poetica è tradito in modo lacunoso e ha dato vita ad un acceso dibattito:

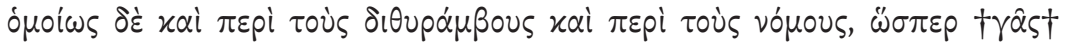

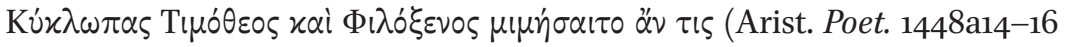
Kassel, Oxford 1965). ${ }^{83}$

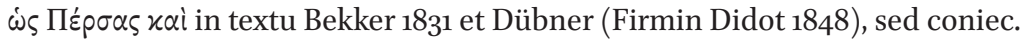
Fr. Medici (iuxta Hardy) vel Petrus Victorius (iuxta Christ, Teubner 1882).

Pazzi aveva tradotto: Pari ratione et qui per dithyrambos et mimos ut fatuos et Cyclopas, Timotheus et Philoxenus utique imitantur (Pazzi 1536, c. 7r).

79 Britann. In Pers. 2,10 p. 95,4-8 Rossetti 2017. 'E BULLET ho emendato questo passo, perché mentre tutti i codici, vuoi per i danni del tempo o per colpa dei copisti, hanno la lezione 'ebulliat', ho ritenuto che si debba leggere 'ebullet' e non 'ebulliat', in quanto 'ebullio' ha la seconda sillaba lunga e si scrive con due 'll', come fa in altri passi Persio: Summa rursus non bullit in unda. In questo passo, se si adotta la lezione 'ebulliat', si dovrebbe abbreviare la sillaba' (trad. di chi scrive). Si è adottato il testo di Rossetti 2017, verificato sull'edizione del commento curata da Takács-Tuhári 2015, 158, su cui vedi le riserve di Muecke 2017.

8o Le moderne edizioni non tengono conto dell'emendamento di Britannico, discusso invece da alcuni umanisti successivi come Ascensius e Nebrija: vd. Rossetti 2017, XCII-XCIII.

81 Altri emendamenti al testo di Persio sono esaminati da Rossetti 2017, XCII-XCIV.

$82 \quad$ Sull'episodio vd. Bionda 2015, LXXI-LXXV.

83 'Lo stesso si può dire a proposito dei ditirambi e dei nòmi: dove i personaggi possono essere [diversamente] rappresentati, come fecero ad esempio, <... e Ar $>$ ga $<$ nei loro ... $>$, Timoteo e Filosseno nei loro Ciclopi' (trad. di Valgimigli 1973). 


\section{Robortello commenta:}

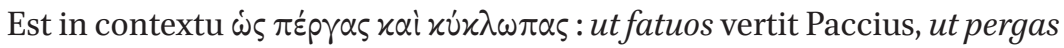
vertit Valla. Nam in dictione $\mu$ ínous agnovit erratum viditque esse legendum vópous. Sed quae amentia est, Dii boni, dicere leges pergas? Quis unquam de his mentionem fecit? Aut quae sunt? Aut quomodo ita potest concinnari hic contextus? $\pi \varepsilon$ ép $\alpha \varsigma$ autem significare fatuos homines, uti vertit Paccius, nusquam legi apud Graecos, neque puto quempiam doctum agnoscere hoc verbum Graecum. Quare legendum est, ut est in

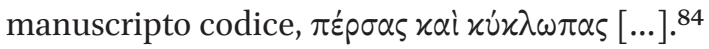

Robortello propone un emendamento, oggi accolto da alcuni editori, e afferma che si tratti di una lezione da lui ritrovata in un manoscritto della Poetica: la cosa è improbabile perché la tradizione della Poetica dipende solo da due manoscritti, un parigino, BnF, Graec. 1741 e un fiorentino, Riccard. $46 .{ }^{85}$ Tuttavia, le edizioni critiche della Poetica non attribuiscono questo emendamento a Robortello, ma a Francesco Medici, un intellettuale schivo e appartato, morto poco prima della pubblicazione del commento di Robortello (1548) e amico di Pier Vettori, l'avversario di Robortello. Le ragioni di questa attribuzione emergono da una lettera inviata al Vettori da un suo allievo, Francesco Spini, che aveva avuto modo di frequentare le lezioni fiorentine sulla Poetica di Robortello (Bionda 2015, LXXIII):

[...] messer Antonio del Camp. [sic], nel ragionarne seco, s'è lasciato uscir di bocca qualche luogo d'importanza, com'egli m'ha confessato pochi giorni sono: ché uno è quella bell'emendazione di $\pi \dot{p} p \gamma \alpha \varsigma$ in $\pi \dot{e} p \sigma \alpha \varsigma$, e quei duo versi d'Empedocle che son nell'ultimo sì laceri, che gl'ha mostro dov'e' sono in Ateneo et in Simplicio [...].

Dalla lettera sembrerebbe che la lezione fosse stata riferita a Robortello da un suo allievo, tal messer Antonio del Camp. (?), personaggio di cui non abbiamo

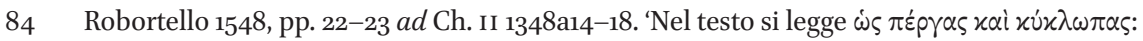
Pazzi ha tradotto ut fatuos, Valla ut pergas. Nella lezione $\mu$ ínovs ha riconosciuto l'errore e si è reso conto che bisognasse leggere vónous. Ma che follia, santi numi, leggere leges pergas? Chi ha mai fatto alcuna menzione di tali leggi? Oppure ha detto quali esse siano? $\mathrm{O}$ in che modo possa quadrare questo testo? Io non ho mai letto in autori greci che $\pi \dot{e} p \gamma \alpha \varsigma$ significhi fatuos, come traduce Pazzi, né ritengo che alcun erudito ammetta l'esistenza di

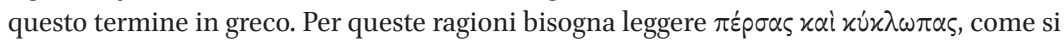
legge su un manoscritto' (trad. di chi scrive).

85 Vd. Kassel 1965, V-IX. 
altre notizie se non questa, secondo cui sarebbe stato un conoscente del Medici e del Vettori. Robortello se ne sarebbe impossessato e, come dice ancora lo Spini, non sembrava disposto a riconoscere l'altrui paternità dell'emendamento: "[...] colui (scil. Robortello) non gnen'ha obbrigo alcuno, ma dice agl'altri d'avergli trovati in più altri autori”. È verisimile che nel commento Robortello abbia introdotto la notizia del codice per dissimulare il modo in cui fosse venuto a conoscenza dell'emendamento.

Le nuove modalità di lettura appena delineate e le possibilità offerte dalla stampa produssero nell'ambito dei commentari la nascita di un originale tipo di testo, che non si limitava a porre nei margini di un'opera un solo commentario, ma radunava per ogni lemma i commenti di diversi autori, antichi, medioevali e moderni in una sorta di commentario plurimo. L'idea di radunare su un argomento le diverse auctoritates del settore non era sconosciuta al mondo del manoscritto, se pensiamo alla costruzione dei commentari variorum nella tarda antichità o ai casi di accumulazione delle esegesi in manoscritti della Bibbia, come la Glossa ordinaria a partire dal XII secolo, e in manoscritti di diritto. ${ }^{86}$ La novità dei commentari plurimi è rappresentata dalla mise en page dei testi esegetici di questi diversi autori: nei margini del testo sono radunati attorno ad un lemma i diversi commenti, di cui è indicato il nome dell'autore, spesso in forma abbreviata [vd. tavola 1]. A partire dall'ultimo decennio del Quattrocento la stampa estende questo fenomeno anche ai commentari di autori classici. ${ }^{87}$

Uno dei primi umanisti a curare la stampa di volumi che contenevano non solo il proprio, ma anche i commentari di altri autori fu Antonio Mancinelli, ${ }^{88}$ il quale godette di un notevole successo alla sua epoca perché fu tra i primi umanisti a sperimentare le potenzialità della stampa ai fini dell'insegnamento, pubblicando decine di opere che coprivano i diversi livelli di insegnamento del latino. ${ }^{89}$ Tra le opere pubblicate dall'umanista si annoverano commenti plurimi a varie opere o auctores scolastici per eccellenza: Virgilio con i commenti a

86 Per i commentari variorum cfr. Zetzel 1975, 337-338, ma anche il caso del ms. Paris. gr. 2923, che contiene i commenti di Siriano, Sopatro e Marcellino, ed estratti di Porfirio ed

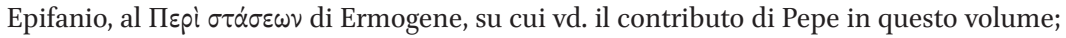
origine e sviluppo della Glossa biblica nel XII secolo sono affrontate da de Hamel 1984, $1-13$.

$87 \quad$ Cfr. Enenkel-Nellen 2013b, 51.

88 Su Mancinelli vd. supra nota 11. Dopo di lui, si inserirono in questo settore della stampa Ascensius e Aldo Manuzio, entrambi grammatici e stampatori (vd. Gehl Humanism for Sale 3.01.5).

89 Cfr. Gehl Humanism for Sale, 3.00, ma anche Mellidi 2007. 
Bucoliche e Georgiche di Servio, Landino e Mancinelli; ${ }^{90}$ Orazio con i commenti antichi dello ps. Acrone e Porfirione e quelli moderni di Cristoforo Landino e dello stesso Mancinelli; ${ }^{91}$ Giovenale con i commenti di Mancinelli, D. Calderini e G. Valla; 92 la Rhetorica ad Herennium con i commenti di F. Maturanzio e suo. ${ }^{93}$ Il successo di queste imprese editoriali è testimoniato dalle loro numerose ristampe nel corso del Cinquecento, quando furono inseriti anche i commenti di altri studiosi. ${ }^{94}$

Tale successo si spiega per varie ragioni: il commentario plurimo rispondeva alle esigenze di docenti e discenti: raccogliendo in un solo libro tutto il sapere 'autorizzato' su un auctor, esso offriva a colpo d'occhio le diverse posizioni della critica su un determinato problema, a partire dagli esegeti più antichi (ad es. Servio per Virgilio, ps. Acrone e Porfirione per Orazio) per arrivare ai più recenti commentari umanistici, come quelli dedicati da Landino o Mancinelli alle opere virgiliane; accanto a ragioni di praticità, che ne facevano un prodotto commerciale, il commentario plurimo corrisponde anche alle nuove modalità di lettura viste in precedenza: offrendo un set di commentatori, esso accontenta un lettore che voglia consultare l'opera ad indicem, alla ricerca di

90 Vergilius, Ecl. e Georg., Comm. di Servio, Cr. Landino e A. Mancinelli, Aen. Comm. di Servio e C. Landino (Venezia, Philippus Pincius, 1491/2, ISTC ivoo188500). In realtà, Mancinelli aveva pubblicato l'anno precedente un proprio commento solo alle Bucoliche e alle Georgiche: Vergilius, Ecl. e Georg., Comm. di A. Mancinelli (Roma, Eucharius Silber, 20.X.1490, ISTC ivoo21950o).

91 Horatius, Opera, Comm. di ps. Acrone, Porfirione, Cr. Landino e A. Mancinelli (Venezia, Philippus Pincius, 28.II.1492/3, ISTC ihoo4550oo).

92 Iuvenalis, Satyrae, Comm. di A. Mancinelli, D. Calderini e G. Valla (Venezia, Johannes Tacuinus de Tridino, 2.XII.1492, ISTC ijoo6620oo). In questo caso Mancinelli colloca intenzionalmente al primo posto il suo commento per dare conto degli interventi che ha apportato al testo tradito, come dichiara nella prefatoria di dedica al veneziano Nicola Rossi: Animadverterent praeterea locis me quam plurimis superiorum sententiis non haesisse, neque alienis demum institisse vestigiis. Qua de re priusquam meis ab interpretationibus divertant, consulto opus erit. Su questo aspetto della stampa giovenaliana cfr. Gehl Humanism for Sale, 3.0. 3.

93 Rhetorica ad Herennium, comm. di F. Maturanzio e A. Mancinelli (Venezia, Philippus Pincius, 8.viI.1496, ISTC icoo6830oo).

94 Vd. ad es. l'edizione virgiliana stampata a Venezia, presso L. Giunta, nel 1533 (CNCE 29507), che aggiunge i lavori esegetici di A. Dati, F. Beroaldo e P. Valeriano, come si legge sul frontespizio: $P$. Virgilii Maronis, poëtarum principis, Opera accuratissime castigata et in pristinam formam restituta, cum acerrimi iudicii virorum commentariis, Seruii, Donati, Mancinelli, Probi, Domitii Calderini atque Ascensi. Quibus acutissime adnotationes sunt additae Christophori Landini, Augustini Dathi et Philippi Beroaldi. Accesserunt insuper ex veterum codicum collatione castigationes et varietates Virgilianae lectionis, per eruditissimum virum Ioannem Pierium Valerianum, olim excerptae, nunc vero iterum ab eodem recognitae. Cum indice copiosissimo [...]. 
risposte a singoli interrogativi. I commentari plurimi rappresentano un forte indebolimento del concetto di autorialità del commento, perché al testo non si affianca il commentario di un solo studioso ben riconoscibile (Servio, Landino o Beroaldo), ma è l'editore-stampatore che diviene in qualche modo l'auctor di questo tipo di volume, riducendo in pezzi i testi dei vari commentatori che stampa nei margini in relazione al lemma cui si riferiscono. ${ }^{95}$

Questa ascesa dello stampatore al rango di autore è resa possibile dall'arrivo in tipografia alla fine del Quattrocento di alcuni umanisti, come Giovanni Britannico, Manuzio o Ascensius, i quali seppero riunire in sé le figure dell'insegnante di grammatica, del commentatore e dello stampatore, ovvero da insegnanti come Antonio Mancinelli che collaborarono a stretto contatto con gli stampatori nella realizzazione delle proprie opere. Nei casi di commentari plurimi l'autorialità veniva in qualche modo rivendicata nelle prefazioni, che forniscono utili informazioni sulle ragioni che portarono alla stampa di questo tipo di commentari. ${ }^{96}$ Mancinelli, ad esempio, in una sorta di dialogo immaginario con Orso Orsini, dedicatario del suo commentario plurimo alle Bucoliche e Georgiche, nonché rettore dello Studium Urbis, giustifica l'aggiunta di un proprio commento accanto all'auctoritas di Servio e al recente lavoro di Landino in virtù degli interventi testuali e delle novità esegetiche da lui proposti:

Diceres autem: "Nonne et Servius iam pluribus annis Vergilii summus habetur interpres, et anno ab hinc secundo Landinus vir profecto eruditissimus? Quid igitur tua eruditione opus?" [...]. Ea quidem omnia ita fore non dubito [...], nec ego eo consilio commentarios edidi, ut homines eruditos redarguam, quamquam aliud alii videtur. Sed uti Vergilii carmen et facilius et apertius habeatur. Eo enim studio, opera, vigilia, diligentia, cura sum usus tum corrigendis textibus, tum illis interpretandis, ut mea

95 Escludendo i commentari variorum tardo-antichi, il commentario plurimo di un autore latino è difficilmente immaginabile nella realtà medioevale del codice, in cui esisteva un limitato mercato librario e per realizzare un progetto editoriale così complesso bisognava avere a disposizione copisti particolarmente competenti e la disponibilità di testi dei diversi commentatori da assemblare. Diverso è il caso di discipline non solo scolastiche, ma con una ricaduta professionale e un vantaggio sociale o economico, come il diritto, in cui non solo docenti e studenti avevano bisogno di avere a disposizione le diverse interpretazioni di un luogo, ma anche chi svolgeva una professione collegata al diritto. In proposito, è assai utile vedere gli sforzi dei copisti per inglobare la Glossa nella pagina del testo biblico, descritti da de Hamel 1984, 14-27.

$96 \quad$ Le dediche e le epistole Lectori forniscono utili informazioni sulla consapevolezza che i commentatori avevano del genere: esse non sono ovviamente elementi specifici del commentario, e solo la seconda è connessa al fenomeno della stampa: Genette 1989, 115-140 e 234-289, distingue giustamente tra dedica e prefazione. 
cum praecedentibus conferentes, necessaria potius quam supervacanea fuisse advertant (A a ii). ${ }^{97}$

Accanto agli elementi che il commentario umanistico e la stampa aggiungono rispetto alla tradizione medievale, esistono anche sezioni che tendono a scomparire nei nuovi prodotti, come l'Accessus ad auctorem, un elemento introdotto nel Medioevo sulla base del commento tardo-antico di Servio a Virgilio e assai frequente nei commentari medievali. ${ }^{98}$ A questo paratesto, che racchiudeva notizie piuttosto approssimative sull'autore e sull'opera commentata o sulle ragioni che ne giustificavano un commento, si preferiscono altre soluzioni, che tengono distinte le varie sezioni dell'Accessus: le lettere di dediche e poi le epistole Lectori forniscono informazioni sulle ragioni che avevano indotto a stampare il commento; spesso una Vita (vd. infra) e un paragrafo dedicato al genere letterario dell'opera commentata precedevano il commento vero e proprio. Di queste scelte, che mettevano in soffitta l'Accessus, si mostra consapevole l'umanista Antonio Costanzi (1436-1490), il quale nell'Argumentum premesso al suo commento dei Fasti di Ovidio ${ }^{99}$ manifesta il proprio fastidio verso il genere dell'Accessus medievale che, a suo dire, ripeterebbe solo cose note e farebbe perdere tempo a studiosi come lui, che oberati da impegni privati e pubblici, possono dedicare poco tempo allo studio:

Autoris vita, librorum numerus et alia quaedam in exponendis autoribus considerari solita ita manifesta sunt, ut consulto a nobis praetereantur studentibus brevitati, quos tantum in modum et privata impediunt

97 'Tu potresti dire: "Non è forse considerato Servio già da molti anni il maggiore interprete di Virgilio? E non c’è da due anni ormai il lavoro di Landino, un uomo veramente coltissimo? Che bisogno c'è (), allora, della tua cultura? [...]". Non metto in dubbio che queste opere siano tali $[. .$.$] , né io ho pubblicato questi commenti con l'intenzione di criticare$ uomini tanto colti, per quanto c'è chi ha un'opinione chi un'altra, ma affinché si abbia a disposizione con più facilità e maggiore chiarezza la poesia di Virgilio. A questo scopo ho messo passione, opera, tempo, attenzione, scrupolo a correggere quei testi, ad interpretarli, di modo che i lettori, confrontando i miei commenti con quelli precedenti, si rendano conto di come siano i miei commenti necessari piuttosto che inutili' (trad. di chi scrive).

98 Sull'Accessus vd. ancora Quaine 1945 e Glauche 1980.

99 Sulla vita e le opere di Costanzi vd. Toscano 2017, 37-53. Del commento ai Fasti di Costanzi esiste l'edizione a stampa, pubblicata a Roma da Eucharius Silber il 23.X.1489 (ISTC iooo17500o) e il manoscritto di dedica a Federico da Montefeltro (Vat. Urb. Lat. 36o): Toscano 2017, 63-82. 
negotia et Fanensis respublica sibi vendicat ut nihil otii fere ad haec studia relinquatur. ${ }^{100}$

La progressiva disintegrazione dell'Accessus produce la nascita di un paratesto indipendente, dedicato alla Vita dell'autore commentato: nel corso del Medioevo la tradizione biografica si era venuta accrescendo della più svariata aneddotica, gonfiando di elementi inverosimili il genere che già nel corso dell'Antichità aveva accolto generosamente aneddoti prodotti per autoschediasma. Di recente è emerso che nel corso del secondo '400 alcuni umanisti presero le distanze dalla tradizione medievale delle Vitae degli scrittori antichi pubblicando solo biografie di provata tradizione antica. ${ }^{101} \mathrm{Il}$ fenomeno si osserva, ad esempio, per la Vita di Giovenale premessa da Domizio Calderini al suo commento: ${ }^{102}$ nel corso del Medioevo la tradizione biografica sul poeta satirico era divenuta debordante a fronte di una estrema povertà di notizie certe; l'umanista reagisce contro tale tendenza tagliando i ponti con questo corpus aneddotico e pubblicando una brevissima vita antica, che egli stesso presenta nel manoscritto e nell'incunabolo con caratteri epigrafici, quasi a volerne indicare l'origine archeologica: EX ANTIQVORVM MONVMENTIS. ${ }^{103}$ In realtà, la Vita scelta da Calderini non aveva un'origine epigrafica, ma era trasmessa da manoscritti di XI-XIII secolo (la cosiddetta Vita Ia di Dürr): essa si allontana dalle altre per la limitazione dell'elemento aneddotico che era sorto attorno alla figura di Giovenale. ${ }^{104}$

100 A. Costanzi, Argum. in Fastos, p. 257,6-9 Toscano, 'La vita dell'autore, il numero dei libri e altre cose del genere, di cui di solito si tiene conto nell'esporre gli autori, sono così noti che sono stati da noi evitati mirando ad essere succinti. D'altronde, gli affari privati ci fanno da impedimento e l'amministrazione della città di Fano ci impegna talmente che non resta quasi più alcuno spazio di tempo da dedicare a questi studi' (trad. di chi scrive).

101 Gli atti del convegno, tenutosi a Roma nel 2013, sono stati pubblicati da Pade 2015: le varie relazioni hanno evidenziato un generale sospetto degli umanisti verso la tradizione biografica ereditata dal Medioevo e la tendenza a sfrondarla degli elementi inverosimili o ad avvalorare notizie che trovavano conferme o nelle opere degli autori stessi o in quelle di altri autori.

102 I dati su questo commento sono supra, 171 e nota 57.

103 Su questa vita e sulla tradizione delle Vitae di Giovenale cfr. Abbamonte 2015. Prima di Calderini, Sicco Polenton aveva dedicato a Giovenale una vita lunghissima e basata sull'aneddotica.

104 Vd. Abbamonte 2015, 205-208. Nel commento all'Ibis di Ovidio, Calderini dichiara di non riportare la vita del poeta, troppo nota (Roma, Sachsel et Golsh, 7.IX.1474, ISTC ic00040000): De Ovidii vita nihil a nobis in hoc loco scribendum est, quom et a multis ea tradita sit et ex poetae libris facile cognosci posset ( $\mathrm{a} \mathrm{i}=$ Rossi 2001, 50). Tuttavia, Calderini si sofferma sull'episodio dell'esilio avvalorando la tesi di una relazione tra Ovidio e la figlia di Augusto. 


\section{Gli elementi interni: il canone degli autori, i nuovi argomenti e i metodi dell'interpretazione}

L'esame dei cosiddetti elementi esterni ha già permesso di toccare aspetti relativi al contenuto dei commentari umanistici (ad es., gli emendamenti ai testi degli autori antichi). Tra le altre novità che riguardano il contenuto si deve enumerare l'ampliamento della selezione degli autori commentati rispetto al Medioevo. ${ }^{105}$ In linea generale, l'interesse didattico persiste nell'Umanesimo e la maggior parte dei commentari riguarda autori presenti nei canoni scolastici medievali; tuttavia, nel corso del Quattrocento furono interessati da un'attività di commento anche autori che erano entrati da poco nei programmi scolastici o che restarono estranei al mondo della scuola. ${ }^{106}$ La scelta di commentare i testi di autori rari non era improntata ad un raffinato esclusivismo, ma rispondeva a nuove esigenze nello studio dei testi antichi: la conoscenza del latino non era più funzionale al solo apprendimento di una lingua franca e strumentale al lavoro che si svolgeva; i testi latini sono ora letti e studiati sia per appropriarsi dei segreti più reconditi della lingua di Roma, che si vogliono riprodurre emulando gli antichi, ${ }^{107}$ sia perché in quei testi sono spesso illustrati fenomeni storici, scientifici o culturali dell'Antichità di cui gli umanisti erano avidi - da questa idea scaturirà il concetto ottocentesco di Altertumswissenschaft, cioè di una scienza che studi tutti gli aspetti dell'Antichità.

A questo scopo si leggono testi di botanica, zoologia, geografia, ecc., ché anzi gli umanisti più raffinati cominciano a ritagliarsi un proprio campo di specializzazione nello studio dell'Antichità: ad esempio all'interno dell'Umanesimo romano diversi intellettuali cominciarono ad interessarsi ad autori poco studiati di età imperiale portandoli perfino nelle aule della scuola. Così, Perotti era considerato alla sua epoca l'esperto di Marziale e delle Silvae di Stazio ${ }^{108}$ e per questa ragione i commentari dedicati da Calderini a questi autori furono considerati nella cerchia romana un'indebita invasione di campo, donde la nascita di un odio reciproco e di una dura polemica tra i due. ${ }^{109}$ Pomponio

105 Gli elenchi di autori letti tra X e Xv secolo sono in Black 2001, 172-274.

106 Enenkel-Nellen 2013b, 23-26, menzionano Vegezio (De re militari), Frontino (Strategemata e De aquae ductibus), Varrone, Catone, Palladio, Columella, Pomponio Mela, Plinio il Vecchio, gli epitomatori Floro e Aurelio Vittore, Claudiano, Ausonio, Orosio, Eutropio, gli Agrimensores e i Geographi minores.

107 Sulle diverse comunità di parlanti latino nel Quattrocento, tra cui vanno inseriti gli umanisti, vd. Ramminger 2015-2016.

108 Su Perotti e Marziale vd. Pade 2008, sul suo interesse per le Silvae vd. Abbamonte 1997.

109 Sulle polemiche di Calderini vd. Campanelli 2001. Forse, va letta in questa prospettiva anche la polemica di Calderini con Angelo Sabino sul testo di Giovenale: Calderini aveva tenuto dei corsi su questo autore, su cui stava lavorando anche Sabino. 
Leto riprese dal suo maestro, Pietro Odo da Montopoli, l'interesse per i Punica di Silio Italico che trasmise al suo allievo Pietro Marso, ${ }^{110}$ mentre egli fu il primo commentatore del De lingua Latina di Varrone, ${ }^{111}$ del decimo libro di Columella ${ }^{112}$ e dei testi della cosiddetta Appendix Vergiliana: anche in questo caso Calderini provò a fargli concorrenza su Silio e sull'Appendix Vergiliana. ${ }^{113}$ Ancora Perotti ed Ermolao Barbaro lavorarono sul testo di Plinio il Vecchio. ${ }^{114}$

Un caso interessante per le ricadute che ebbe sulla storia culturale europea è costituito dalla storia dei commentatori della Poetica di Aristotele, un testo di cui non esisteva una tradizione esegetica medievale e quattrocentesca in oriente, né in occidente: dopo due traduzioni latine (Giorgio Valla nel 1498 e Alessandro Pazzi nel 1536), i primi commenti furono scritti nel giro di poco più di un decennio da Robortello (nel 1548) e Vettori (nel 1560). L'effetto di questi lavori è che l'opera aristotelica attirò l'attenzione non solamente degli specialisti; si produssero volgarizzamenti ed altri commenti in latino e nelle lingue

110 Lo studio e l'insegnamento di Silio Italico fu introdotto a Roma alla metà del Quattrocento da Pietro Odo da Montopoli: cfr. Donati 2000, 25, 60-61. Sullo studio di Silio nella Roma del xV secolo vd. Bassett-Delz-Dunston 1976 e l'introduzione a Dunston-Muecke 2011. La princeps di Silio fu edita da Leto (Roma, stampatore di Silio, 1471, ISTC isoo50400o), mentre il commento di Pietro Marso a Silio fu stampato a Venezia, Baptista e Tortis, 6.v.1483, ISTC isoo50700o.

111 Varro, De lingua Latina, ed. P. Laetus [Roma, G. Lauer, 1471-1472], ISTC ivooog40oo: sugli studi dedicati da Leto a Varrone vd. Accame Lanzillotta 1998 e Accame Lanzillotta 2008, 124-137.

112 La princeps del decimo libro di Columella fu probabilmente curata da Leto (Roma, medesimo stampatore del Silio Italico, 1471, ISTC icoo76270o), mentre il commento a quel libro di Columella, l'unico in versi, fu opera di Leto: Venezia, stampatore del De officiis di Cicerone, c. 1481-1482, ISTC icoo763250.

113 Il commento di Leto ai testi dell'Appendix si conserva in una redazione manoscritta sui seguenti testimoni (cfr. Abbamonte 2012, 131-133): Vat. Lat. 3255, Oxford, Canon. Class. Lat. 54, Roma, Corsiniano 1839 (43.F.21). La princeps si trova nel primo volume dell'intero commento di Leto alle opere di Virgilio, stampato in due volumi a Brescia da Boninus de Boninis, 1487-149o?, ISTC ilooo2330o: cfr. Abbamonte 2012, 129-130. Il commento di Calderini ad alcuni testi dell'Appendix fu stampato postumo a Milano, Simon Magniagus?, c. 1480, ISTC icooo03900o: sui rapporti tra i due testi vd. Rossetti 2013.

114 L'interesse verso il testo di Plinio produsse quella che Enenkel-Nellen 2013b, 26, definiscono brillantemente una lettura Plinyized del mondo antico, che tende a spiegare ogni evento culturale, naturale, tecnologico e scientifico alla luce della Naturalis historia di Plinio. Il fenomeno riguarda anche il mondo moderno: nel suo diario di bordo Cristoforo Colombo cita spesso Plinio e legge i fenomeni naturali del Nuovo mondo con gli occhi di Plinio (Abbamonte 1999); la Naturalis historia era studiata anche dai futuri missionari cattolici, come dimostra il numero di copie presenti nella Biblioteca Apostolica Vaticana che riportano la segnatura della congregazione missionaria Propaganda fide (ediz. Roma 1470 Prop. Fide II.225 Roma 1473 Prop. Fide II.224; Treviso 1479 Prop. Fide II.98 e IV.240; Venezia 1487 Prop. Fide III.96; Venezia 1491 Prop. Fide III.120; Bologna 1493 Prop. Fide IV.170). 
volgari: in breve, la Poetica contrastò il dominio dell'Ars poetica di Orazio nel campo delle teorie sulla poesia e fu al centro di un dibattito europeo sulle forme della poesia drammatica e del poema epico, che vide tra i suoi protagonisti Torquato Tasso.

Nei casi di testi scientifici, i commentatori umanistici non si limitavano ad osservazioni sulla lingua latina, ma portavano avanti ricerche che preannunciano il metodo della scienza empirica moderna: essi si sforzavano di individuare i Realien cui fanno riferimento i testi antichi. In un primo momento questi tentativi furono condotti non tanto attraverso l'osservazione naturalistica, ma attraverso il confronto con altri testi antichi. Così, nel suo commento al decimo libro dell'opera di agricoltura di Columella, Leto si sforza di identificare le numerose piante da giardino ivi menzionate attraverso il confronto con le opere botaniche di Teofrasto, che erano state tradotte per la prima volta in latino in quegli anni dal suo amico Teodoro Gaza, o attraverso il confronto con altre opere latine sullo stesso tema, ad es. il trattato De re rustica di Varrone, i libri botanici di Plinio il Vecchio o le Georgiche di Virgilio con il commento di Servio. ${ }^{115}$ Analogamente, a partire dalla metà del '400 si produsse a Roma una serie di commenti ai Fasti di Ovidio, che offrivano una lettura non solo scolastica dell'opera, quale si osserva nei pur meritori commenti al poema ovidiano elaborati dalla scuola di Orléans (x secolo). I commentatori umanistici mostrano una nuova sensibilità verso i temi 'antiquari' della città di Roma, che divennero sempre più il centro degli interessi degli intellettuali che si radunavano attorno a Pomponio Leto e poi della cultura antichistica dell'Urbe nel corso del Cinquecento. ${ }^{116}$

Le diverse finalità con cui gli umanisti guardano alla lingua e alle opere di Roma producono un approccio differente anche in quegli aspetti di analisi linguistica che erano i più comuni del genere del commentario sin dall'Antichità e nel corso del Medioevo. Si assiste nel Quattrocento ad una rielaborazione di due concetti presenti in tutte le stagioni dei commentari, quelli di imitatio ed

115 Sia consentito il rimando ancora ad Abbamonte 2012, 183-199.

116 Sulla lettura dei Fasti nel Medioevo cfr. Toscano 2017, 8-15. La fortuna dei Fasti nell'Umanesimo, soprattutto romano, è esaminata da Fritsen 2015 e Toscano 2017, 15-34. Produsse un commento ai Fasti di Ovidio, Pietro Odo da Montopoli, il maestro di Pomponio Leto, che tenne un corso sui Fasti conservato nel manoscritto Vat. Lat. 1595; Leto trascrisse il manoscritto dei Fasti Vat. Lat. 3264, inserendovi note di argomento antiquario. Nel 1482 Paolo Marsi, allievo di Leto, pubblicò il primo commento a stampa dell'opera (Venezia, Baptista de Tortis, 24.XII.1482, ISTC iooo170ooo), seguito nel 1489 da Antonio Costanzi (Roma, E. Silber, 23.X.1489, ISTC iooo175000). Inoltre, Domizio Calderini ha lasciato appunti sui Fasti nel manoscritto München, Bayerische Staatsbibliothek, Clm 754, mentre del commento di Maturanzio, allievo e segretario di Perotti, oggi perduto dà notizia Paolo Marsi (f. a i): cfr. Toscano 2017, 20-21. 
aemulatio. ${ }^{117}$ Nella tradizione scolastica a partire da Prisciano, per tutto il corso del Medioevo fino alla piena età moderna, l'aemulatio era un tipo di esercizio che il docente illustrava già nel corso dei primi anni e che si continuava a svolgere anche nella fase più avanzata del commento soprattutto nell'ambito della parafrasi. I testi latini, soprattutto in versi, hanno sempre avuto bisogno di una parafrasi sia per la loro difficoltà lessicale sia per quella relativa alla costruzione delle frasi. Per superare l'ostacolo lessicale, il commentatore glossava i termini o le espressioni ritenute complesse con forme più semplici, mentre la parafrasi aiutava a ricomporre la struttura del testo latino in una sequenza comprensibile. Allo studente era richiesto di riformulare la frase dell'auctor individuandone il soggetto, il verbo e le diverse reggenze del verbo; quest'esercizio tendeva a riprodurre il cosiddetto ordo naturalis del testo e nei documenti medievali va spesso sotto il nome di positio verborum. ${ }^{118}$ Accanto a queste due operazioni di glossa e parafrasi, lo studente doveva saper svolgere un altro esercizio che consisteva nel riprodurre in latino con parole proprie il testo commentato: questo esercizio di imitazione e competizione con il testo-base va sotto il nome di aemulatio. ${ }^{119}$

L'aemulatio, relativa alla positio verborum, divenne all'inizio del Cinquecento un esercizio prescrittivo che imponeva la riproduzione di testi nello stile di Cicerone (il cd. Ciceronianismo, promulgato già nel corso del '40o, ad es., dai Barzizza): contro questo modello unico del latino classico scritto si schiereranno vari umanisti (Lorenzo Valla, Beroaldo il Vecchio con l'intero umanesimo bolognese e soprattutto Erasmo). Accanto a questo tipo di aemulatio, il termine assume nei commentari umanistici anche un altro significato, passando dal didattico esercizio di stile ad un concetto più vicino alla nascente critica letteraria: per alcuni umanisti aemulatio definisce il rapporto tra un passo del testo commentato ed uno dello stesso autore o di un altro. Essa comprende

117 Fondamentale per uno studio del concetto di imitatio nell'Umanesimo il saggio di McLaughlin 1995, in part. 98-125 sul concetto di imitazione negli umanisti che si dedicarono all'insegnamento (Gasparino Barzizza, Antonio da Rho e Guarino Veronese).

118 Su questa parte dell'insegnamento grammaticale vd. Black 2001, 70-73 e ad indicem; Enenkel-Nellen 2013b, 37-38.

119 L'importanza di questo esercizio è così riassunta da Enenkel-Nellen 2013b: "Due to the importance of paraphrase in the educational system, this instructional practice lodged itself in the flesh and blood of all late medieval and early modern commentators. In other words, it became an entrenched habit, a standard modus cogitandi, legendi et meditandi. The late medieval and early modern scholar was always tempted to paraphrase a text first - for he considered paraphrasing the best way to appropriate it definitively" (38). McLaughlin 1995, 99, riporta una distinzione tra imitatio ed aemulatio stabilita da Antonio da Rho: Imitatio simplex est et livorem atque invidiam non admittit. Æmulatio autem habet imitandi studium sed cum malitiae operatione. 
l'individuazione dei loci paralleli, ma non si limita ad essa, in quanto l'aemulatio può implicare che il passo commentato emuli l'altro, cioè che l'autore commentato abbia letto e adoperato il passo che emula in un rapporto di intertestualità con il suo modello. Di seguito, tre esempi dell'uso di questo concetto da parte di Britannico e Parrasio:

VIDE SIS NE MAIORUM aemulatio est Horatii: "O puer ut sis Vitalis metuo et maiorum nequis amicus Frigore te feriat” (Serm. 2,1,6o-62).

Britannico, In Persium 1,108, p. 80,8-9 Rossetti 2017 = Takács-Tuhári 2015, 148

Dextro hercule [...] Aemulatio est Horatii: "Dives amico / Hercule" (Serm. 2,6,12-13).

Britannico, In Persium 2,11-12, p. 96,1 et 9 Rossetti 2017 = Takács-Tuhári 2015, 159

IAM MiHI CERnUntur Aemulatio Callimachi qui eadem se per furorem

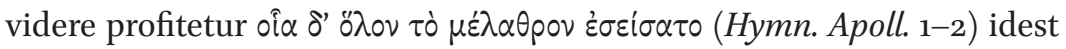
"en aedes ut tota tremit". 120

Parrhas., Comm. in Claud. De raptu Proserpinae, 7 , Venezia, J. et B. Rubeus Vercellensis, c. 1510, ISTC icoo70800o, f. a ii

Britannico ricorre a questo termine nel suo commento per stabilire richiami di Persio al testo di Orazio ${ }^{121}$ e in un caso a quello di Terenzio: ${ }^{122}$ gli insistiti riferimenti ad Orazio potrebbero indicare una linea interpretativa di Britannico, secondo cui il linguaggio di Persio avrebbe avuto precisi confronti con quello del suo predecessore nel genere della satira. ${ }^{123}$

120 'BADA CHE TU NON SIA Aemulatio di Orazio: Ragazzo mio, temo proprio che tu non abbia vita lunga e che qualcuno dei grandi, oggi tuo amico, non abbia a ferirti con la sua freddezza' (trad. di chi scrive). 'CON L'AIUTO Di ERCOLE Aemulatio di Orazio: Ricco grazie ad Ercole amico' (trad. di chi scrive). 'GIÀ MI SI mostrano Aemulatio di Callimaco che dichiara di vedere le stesse cose in uno stato di esaltazione: Come trema tutto l'altare, cioè Ecco che trema tutto l'altare' (trad. di chi scrive).

121 L'espressione ricorre nel commento di Britannico in riferimento ad Orazio nei seguenti luoghi (si riporta qui e nella nota successiva) solo la numerazione di Rossetti 2017: Pers. 1,108 (p. 8o,8-9 Rossetti); 2,9-10 (p. 95,2-4 Rossetti); 2,11-12 (p. 96,9 Rossetti); 3,8 (p. 119,11 Rossetti); 3,83 (p. 146,3-4 Rossetti); 3,97 (p. 148,12-13 Rossetti); 4,11-12 (p. 159,11 Rossetti); 4,14 (p. 161,1 Rossetti); 4,34 (p. 168,11-12 Rossetti); 5,4 (p. 178,17-19 Rossetti); 5,54 (p. 194,1516 Rossetti); 5,153 (p. 223,2-3 Rossetti); 5,161 (p. 224,10 Rossetti); 5,161 (p. 224,12-13 Rossetti); 6,19-20 (p. 251,14-15 Rossetti); 6,23 (p. 253,4-5 Rossetti); 6,69 (p. 269,12-13 Rossetti); 6,71 (p. 270,3-5 Rossetti).

122 Cfr. Britannico, In Pers. 5,169 (p. 226,6-7 Rossetti).

123 I dati sono presentati e discussi in Rossetti 2017, XCV. 
Talvolta, questo accostamento prende il nome di imitatio, come si osserva in un passo del commento di Calderini all'Ibis:

ORA MUltiplicata Hyperbole est Homerica, cum esset descripturus numerum navium: eam imitatus est Hostius in secundo belli Istri: "Non mihi si linguae Sint centum atque ora sint totidem" (frg. 3 Blänsdorf, in Macr. Sat. 6,3,6). Et demum Virgilius: "Non, mihi si linguae centum" (Georg. 2,43; Aen. 6,625).124

Calderini In Ibim 204, f. b ii = Rossi 2001, 64

Il termine imitatio sembra avere un significato analogo ad aemulatio anche nei seguenti passi tratti da commenti di Calderini e Leto:

Iuvenalis Lucillii acerbitatem puritate Horatiana quam maxime adamavit et imitatus est.

Calderini In Iuv. Praef. a Giuliano de' Medici, Roma 1.IX.1474, ISTC icooo36ooo, f. a iii)

"Sic me servavit Apollo" (Hor. Serm. 1, 9, 78), idest iudices ad statuam Apollinis sedentes, ad quos tractus est Bolanus. Non ignoro tamen Porphyrionem ex imitatione Homerica eum locum interpretatum esse.

Calderini In Iuv. 1,128, f. b.

AMAROR imitatio est Lucretii qui ait : "Denique in os salsi venit humor saepe saporis/ Cum mare versamur propter dilutaque contra./ Cum tuimur misceri absinthia tangit amaror" (4, 222-224).

Laet., In Verg. Georg. 2,247, in Abbamonte 2012, 175-176 $6^{125}$

A differenza di quanto scrive Britannico su Persio, Calderini ritiene che la satira di Giovenale abbia saputo mescolare gli elementi della poesia di Lucilio con quelli di Orazio; inoltre, l'umanista mostra una notevole sensibilità poetica nel segnalare che Orazio, secondo Porfirione, avrebbe concluso la satira dello scocciatore con l'apparizione di Apollo per imitare (in chiave antifrastica) l'intervento di Apollo in Omero. Il commento alle Georgiche di Pomponio Leto,

124 'MOLTiPliCAZIONE Di Voci Iperbole di Omero che sta descrivendo il numero delle navi; l'ha imitata Ostio nel secondo libro della Guerra istriana: Neanche se avessi cento lingue e altrettante bocche, e poi Virgilio: Neanche se avessi cento lingue' (trad. di chi scrive).

125 Sulla princeps del commento virgiliano di Leto vd. supra nota 113. Poco dopo, Leto ritorna sull'origine lucreziana di un passo virgiliano: HABENDO subigendo et tractando et est imitatio Lucretii (Laet. In Verg. Georg. 2,250). 
invece, presenta numerosi rimandi a versi di Lucrezio, considerati alla base di alcune immagini poetiche di Virgilio. Attraverso l'imitatio Leto è il primo a stabilire il collegamento, oggi ampiamente riconosciuto dalla critica, tra il poema didascalico di Virgilio e il De rerum natura di Lucrezio.

\section{$4 \quad$ Conclusioni}

In conclusione, il commentario che si andò sviluppando a partire dalla seconda metà del Quattrocento mantenne intatti numerosi elementi che aveva ereditato dalla tradizione tardo-antica e medievale, ma propose anche elementi di novità. Alcuni di essi furono favoriti dalle nuove possibilità offerte dall'introduzione della stampa: all'apparenza, queste innovazioni si presentano come trasformazioni relative ad aspetti esterni al testo o al suo commentario, ma in realtà l'introduzione degli indici o delle raccolte di animadversiones e la composizione di commentari plurimi implicano un profondo mutamento del contenuto e dei metodi di compulsare questo tipo di testi e presuppongono un nuovo tipo di lettore, che si affianca al consueto pubblico scolastico dei commentari.

A partire dalla seconda metà del Quattrocento videro la luce commenti dedicati anche ad opere che non rientravano strettamente nel curriculum scolastico: ciò permise di creare una tradizione esegetica relativa ad opere all'apparenza minori, come i testi poetici di Marziale, Silio Italico o le Silvae di Stazio. Questo interesse verso poeti minori o autori venuti alla luce da poco diede l'avvio alla ricerca dei loci paralleli e ad un embrione di interpretazione intertestuale (ma anche intratestuale) soprattutto dei testi poetici. In questo periodo, si assiste anche ad un allargamento del canone degli autori inseriti nei programmi scolastici, che include testi scientifici come quelli di Columella o Varrone, la Poetica di Aristotele e letture in chiave antiquaria di testi che pure avevano goduto di una tradizione esegetica, ma prevalentemente grammaticale, come i Fasti di Ovidio.

\section{Bibliografia}

Abbamonte, G. (1997). Ricerche sul commento inedito di Perotti alle Siluae di Stazio. Studi Umanistici Piceni 17, pp. 9-20.

Abbamonte, G. (1999). Some Suggestions for the Italian School Teaching of the History of the 15th Century Geographic Discoveries. Euphrosyne 27, pp. 315-338. 
Abbamonte, G. (2005). Sul codice genetico del Cornu Copiae di Niccolò Perotti: una modesta proposta. Roma nel Rinascimento, pp. 13-17.

Abbamonte, G. (2007). Su alcuni codici Boeziani della Biblioteca Nazionale di Napoli "Vittorio Emanuele" III. In: M. Špelič OFM e P. Limoncini (edd.), Boezio e Gregorio Magno tra Antichità e Medioevo. Atti del Primo Simposio Internazionale di Rapallo, Rapallo (Genova) 31 marzo-2 aprile 2005, Rapallo e Ljubljana, pp. 99-140.

Abbamonte, G. (2012). Diligentissimi uocabulorum perscrutatores. Lessicografia ed esegesi dei testi classici nell'Umanesimo romano di XV secolo. Pisa.

Abbamonte, G. (2015). Materiali biografici antichi su Giovenale recuperati da Domizio Calderini. In: Pade 2015, pp. 177-2016.

Accame Lanzillotta, M. (1998). Le annotazioni di Pomponio Leto ai libri VII-X del De lingua Latina di Varrone. Giornale Italiano di Filologia 50, pp. 41-57.

Accame Lanzillotta, M. (2008). Pomponio Leto. Vita e insegnamento. Tivoli.

Baschera, C., ed. (1999). Gli scoli veronesi a Virgilio. Introduzione, edizione critica e indici a cura di $\mathrm{C}$. Baschera. Verona.

Bassett, E. L., Delz, J. e Dunston, A. J. (1976). Silius Italicus, Tiberius Catius Asconius, in Catalogus Translationum et Commentariorum III, pp. 1-398.

de Beer, S. (2013). The World Upside Down: the Geographical Revolution in Humanist Commentaries on Pliny's Natural History and Mela's De situ orbis (1450-1700). In: Enenkel-Nellen 2013, pp. 139-197.

Bionda, S., ed. (2015). Poetica d'Aristotile tradotta di greco in lingua volgare fiorentina da Bernardo Segni gentiluomo et accademico fiorentino. Roma.

Black, R. (2001). Humanism and Education in Medieval and Renaissance Italy. Tradition and Innovation in Latin Schools from the Twelfth to the Fifteenth Century. Cambridge.

Black, R. (2007). Education and Society in Florentine Tuscany. Teachers, Pupils and School, c. 1250-150o. Leiden.

Black, R. e Pomaro, G. (2000). La consolazione della filosofia nel Medioevo e nel Rinascimento italiano / Boethius' Consolation of Philosophy in Italian Medieval and Renaissance Education. Firenze.

Branca, V. e Pastore Stocchi, M., edd. (1972). Angelo Poliziano, Miscellaneorum centuria secunda. Firenze.

Buck, A. (1975). Einführung. In: Buck-Herding (1975), pp. 7-19.

Buck, A. e Herding, O., edd. (1975). Der Kommentar in der Renaissance. Boppard.

Campanelli, M. (2001). Polemiche e filologia ai primordi della stampa. Le Observationes di Domizio Calderini. Roma.

Desmet-Goethals, M. J. (1975). Die Verwendung der Kommentare von Badius, Mancinellus, Erasmus und Corderius in der Disticha Catonis. In: Buck-Herding 1975, pp. $73^{-88 .}$ 
Devoti, L. (1999). Un rompicapo medievale: l'architettura della pagina nei manoscritti e negli incunaboli del Codex di Giustiniano. In: P. Busonero, M. A. Casagrande Mazzoli, L. Devoti, e E. Ornato (edd.), La fabbrica del codice. Materialiper la storia del libro nel tardo medioevo, Roma, pp. 141-206.

Dunston, J. e Muecke, F., edd. (2011). Domizio Calderini, Commentary on Silius Italicus. Génève.

Enenkel, K. A. (2014). Transformations of the Classics via Early Modern Commentaries. Leiden.

Enenkel, K. A. (2014a). Introduction. The Transformation of the Classics. Practices, Forms, and Functions of Early Modern Commenting. In: Enenkel 2014, pp. 1-12.

Enenkel, K. A. e Nellen, H., eds. (2013). Neo-Latin Commentaries and the Management of Knowledge in the Late Middle Ages and the Early Modern Period (1400-1700). Leuven.

Enenkel, K. A. e Nellen, H. (2013b). Introduction. Neo-Latin Commentaries and the Management of Knowledge. In: Enenkel-Nellen 2013, pp. 1-76.

Ferreri, L., ed. (2012). Aulo Giano Parrasio, De rebus per epistolam quaesitis (Vat. Lat. 5233, ff. $1 r-53 r$ ). Introduzione, testo critico e commento filologico. Roma.

Fritsen, A. (2015). Antiquarian Voices. The Roman Academy and the Commentary Tradition on Ovid's Fasti. Columbus (Ohio).

Frova, C. (1973). Istruzione e educazione nel medioevo. Torino.

Frova, C. (1992). Le scuole municipali all'epoca delle università, in Vocabulaire des écoles et des méthodes d'enseignement au moyen âge, Turnhout, pp. 177-190.

Garin, E. (1957). L'educazione in Europa (1400-1600). Bari.

Gehl, P. F. (2008). Off the Press into the Classroom: Using the Textbooks of Antonio Mancinelli. History of Education and Children's Literature III/2, pp. 19-30.

Gehl, P. F. Humanism for Sale. Humanism for Sale. Making and Marketing Schoolbooks in Italy, 1450-1650, http://www.humanismforsale.org/text/ (ultima consultazione, 27.08.17).

Genette, G. (1989). Soglie. I dintorni del testo. Torino (tit. orig. Seuil, Paris 1987).

Gibson, R. K. e Shuttleworth Kraus, Ch., eds. (2002). The Classical Commentary. Histories, Practices, Theory. Leiden.

Glauche, G. (1980). s.v. Accessus ad auctores, in Lexikon des Mittelalters, Vol. I. Stuttgart, coll. $71-72$.

Grendler, P. (1989). Schooling in Renaissance Italy. Baltimore.

Grendler, P. (1996). Books and Schools in Italian Renaissance. Great Yarmouth, Norfolk.

Häfner, R. e Völkel, M., hrsg. von (2006). Der Kommentar in der Frühen Neuzeit. Tübingen.

de Hamel, C. F. R. (1984). Glossed Books of the Bible and the Origins of the Paris Booktrade. Woodbridge. 
Holtz, L. (1984). Les manuscrits latins à gloses et à commentaire de l'Antiquité à l'époque carolingienne. In: Questa-Raffaelli 1984, pp. 139-167.

Holtz, L. (1985). La redécouverte de Virgile aux VIII ${ }^{\mathrm{e}}$ et IX ${ }^{\mathrm{e}}$ siècles d'après les manuscrits conservés. In : Lectures medièvales de Virgil, ME FR 80, Roma, pp. 9-30.

Holtz, L. (1986). Les manuscrits carolingiens de Virgile. In: M. Gigante (a cura di), La fortuna di Virgilio, Napoli, pp. 125-149.

Iacono, A. (2005). Uno studente alla scuola del Pontano a Napoli: le recollectae del ms. 1368 (T. 5. 5) della Biblioteca Angelica di Roma, edizione critica con introduzione e commento. Napoli.

Irigoin, J. (1984). Livre et texte dans les manuscrits byzantins de poètes. Continuité et innovation. In: Questa-Raffaelli 1984, pp. 85-102.

Jeanneret, M. (1990). Préfaces, commentaires et programmation de la lecture. L'exemple des Métamorphoses. In: Mathieu-Castellani et Plaisance 1990a, pp. 31-39.

Kallendorf, C. (2005). Marginalia and The Rise of Early Modern Subjectivity. In: Pade 2005, pp. 111-128.

Kassel, R., ed. (1965). Aristoteles, Poetica. Oxford.

Kristeller, P. O. (1960). Der Gelehrte und sein Publikum im späten Mittelater und in der Renaissance. In: Medium Aevum vivum. Festschrift f. W. Bulst, Heidelberg, pp. 212-230.

Kristeller, P. O. (1985). Humanist Learning in the Italian Renaissance. In: P. O. Kristeller, Studies in Renaissance Thought and Letters, Vol. II, 93-110 (orig. pubblicato in The Centennial Review 4, 1960, 243-266).

Lazzari, F., ed. (2005). Antonio Mancinelli (1452-1505) pedagogo, grammatico e umanista. Velletri.

Leonardi, C., Morelli, M., e Santi, F., a cura di (1995). Fabula in tabula. Una storia degli indici dal manoscritto al testo elettronico. Spoleto.

Manacorda, G. (1914). Storia della scuola in Italia. Il medio evo. Milano-Palermo.

Maniaci, M. (2002). «La serva padrona ». Interazioni fra testo e glossa sulla pagina del manoscritto. In: V. Fera, G. Ferraù e S. Rizzo (eds.), Talking to the Text. Marginalia from Papyri to Print. Proceedings of a Conference held at Erice, 26 September-3 October 1998, as the 12th Course of International School for the Study of Written Records, Messina, pp. 3-35.

Mathieu-Castellani, G. et Plaisance, M., Textes réunis et présentés par (1990a). Les commentaires et la naissance de la critique littéraire. Actes du Colloque International sur le Commentaire, Paris, mai 1988. Paris.

Mathieu-Castellani, G. e Plaisance, M. (199ob). Avant-propos. In: Mathieu-Castellani et Plaisance 1990, pp. 7-8.

McLaughlin, M. L. (1995). Literary Imitation in the Italian Renaissance The Theory and Practice of Literary Imitation in Italy from Dante to Bembo. Oxford.

Mellidi, C. (2007). s.v. Mancinelli, Antonio, in DB I vol. 68, Roma, pp. 450-453. 
Milde, W. (1982). Zur Druckhäufigkeit von N. Perottis Cornucopiae und Rudimenta grammatices im 15. und 16. Jahrhundert. Studi Umanistici Piceni 2 [= Res Publica Litterarum 5], pp. 29-42.

Most, G. W., ed. (1999). Commentaries - Kommentare. Göttingen.

Muecke, F. (2015). Rec. a Takács-Tuhári 2015. Bryn Mawr Classical Review 2017.11.o9.

Osmond, P. J. (2005). The Valla Commentary on Sallust's Bellum Catilinae: Questions of Authenticity and Reception. In: Pade 2005, pp. 29-48.

Pade, M., ed. (2005). On Renaissance Commentaries. Hildesheim.

Pade, M. (2005a). Introduction. In: Pade 2005, pp. 1-2.

Pade, M. (2005b). Niccolò Perotti's Cornu copiae: Commentary on Martial and Encyclopaedia. In: Pade 2005, pp. 49-63.

Pade, M. (2008). Commenti perottini a Marziale? Il ms. B 131 sup. della Biblioteca Ambrosiana di Milano. Studi Umanistici Piceni 28, pp. 79-95.

Pade, M. (2013). Niccolò Perotti's Cornu copiae: the Commentary as a Repository of Knowledge. In: Enenkel-Nellen 2013, pp. 241-262.

Pade, M., ed. (2015). Vitae Pomponianae: Lives of Classical Writers in Fifteenth-Century Roman Humanism. Rencessanceforum 9.

Pazzi, A. (1536). Aristotelis Poetica per Alexandrum Paccium, patritium Florentinum, in Latinum conversa. Venezia, eredi di A. Manuzio e A. Torresano.

Powitz (1979). Textus cum commento. Codices Manuscripti 5, pp. 80-89.

Quaine, E. A. (1945). The Medieval Accessus. Traditio 3, pp. 215-264.

Questa, C. e Raffaelli, R., a cura di (1984). Il libro e il testo. Atti del convegno internazionale, Urbino 20-23 settembre 1982, Urbino.

Ramminger, J. (2015-2016). Language Chance in Humanist Latin: the Case of traducere (to translate). Analecta Romana Instituti Danici 40-41, pp. 35-62.

Raso, T. (2001). Il «Boezio» Abruzzese del XV secolo. Testo latino-volgare per l'insegnamento della sintassi latina. Edizione critica con studio introduttivo e glossario. L'Aquila.

Rigolot, F. (1990). Introduction à l'étude du "commentaire". L'exemple de la Renaissance. In: Mathieu-Castellani et Plaisance 1990a, pp. 51-62.

Robortelli, F. (1548). Francisci Robortelli In librum Aristotelis de arte poetica explicationes, Firenze, Lorenzo Torrentino.

Rossetti, F. (2013). Il commento di Domizio Calderini all'Appendix Vergiliana. Humanistica 8/1, pp. 131-146.

Rossetti, F. (2017). Il commento di Giovanni Britannico a Persio e la sua ricezione nel Cinquecento europeo. Edizione critica e studio introduttivo. PhD Thesis, Napoli-Strasbourg.

Rossi, L. C., a cura di (2001). Domizio Calderini, Commentarioli in Ibyn Ovidii, Firenze.

Sabbadini, R. (1902). Dei metodi nell'insegnamento della sintassi latina: considerazioni didattiche e storiche. RFIC 30, pp. 304-314. 
Sabbadini, R. (1922). Il metodo degli umanisti. Firenze.

Saitta, G. (1928). L'educazione dell'Umanesimo in Italia. Venezia.

Sautel, J. H. (1999). Essai de terminologie de la mise en page des manuscrits à commentaire. Gazette du livre médiéval 35, pp. 17-31.

Severi, A. (2015). Filippo Beroaldo il Vecchio un maestro per l'Europa. Da commentatore di classici a classico moderno (1481-1550). Bologna.

Takács, L. e Tuhári, A., eds. (2015). Two Renaissance Commentaries on Persius. Bartholomaeus Fontius' and Ioannes Britannicus' Commentaries on Persius. Piliscsaba Budapest.

Toscano, F. (2017). Il commento di Antonio Costanzi da Fano ai Fasti di Ovidio. Edizione critica del commento a Fast. I-III. PhD Thesis, Salerno.

Ulery, R. W. Jr. (2005). Sallust's Bellum Catilinae in the Edition of Venice 1500: the Medieval Commentary and the Renaissance Reader. In: Pade 2005, pp. 7-28.

Valgimigli, M. (1973). Aristotele, Opere, vol. x Retorica e Poetica. Bari.

Wilson, N. G. (1984). The Relation of Text and Commentary in Greek Books. In: QuestaRaffaelli 1984, pp. 103-110.

Zetzel, J. E. G. (1975). On the History of the Latin Scholia. HSCPh 79, pp. 335-354.

Zumthor, P. (1990). La glose créatrice. In: Mathieu-Castellani et Plaisance 1990, pp.11-18.

Zunzt, G. (1938-1939). Die Aristophanes-Scholien der Papyri. Byzantion 13, pp. 631-69o; 14, pp. 545-614. 